\title{
Models for shear properties/behavior of dry fibrous materials at various scales: a review
}

\author{
Elena Syerko • Sébastien Comas-Cardona • Christophe Binetruy
}

\begin{abstract}
During forming operations of dry fibrous materials in order to get the composite preforms, the deformation mode is substantially determined by the occurring shear strains. Consideration of the material shear response namely from the theoretical point of view has gained high importance because multi-scale nature and anisotropy of fibrous materials complicates their experimental investigation, which raises the question of the tests realizability, complexity, and cost. This work analyses and classifies the existing theoretical approaches for the shear prediction with respect to the mathematical principles employed, as well as with respect to the architecture of diverse fibrous materials, to which the approach can be applied. Both classifications depend in turn on the main criterion of classification emphasized here - the scale of observation of the shear load. According to the theoretical framework, the approaches form the main groups based either on purely geometrical reasoning, or on finite elements analysis, or on energy minimization, or on forces equilibrium principle. The approaches are compared according to the total of mechanical factors included in their analysis and typical for a certain stage of shear load and a certain scale (micro-, meso-, or macro-). The advantages and difficulties of each of the methods are evaluated.
\end{abstract}

Keywords Forming · Fabric/textile - Shear property/ behavior · Multiscale modeling - Finite element analysis (FEA) $\cdot$ Energy minimization

E. Syerko · S. Comas-Cardona $\cdot$ C. Binetruy

École Centrale de Nantes, Research Institute in Civil Engineering and Mechanics (GeM), UMR CNRS 6183, 1 rue de la Noë, BP 92101, 44321 Nantes Cedex 3, France

e-mail: olena.syerko@ec-nantes.fr

\section{Introduction}

The formability of composite parts is substantially determined by the mechanical response to loading of the dry reinforcements - fibrous materials. During the forming of composites, the reinforcements undergo high tensile stresses and large strains, and the strains are mainly associated with the in-plane shear mode. In the late stages of forming deformation particularly the shear mode causes the wrinkle apparition. Since investigation of the deformation modes before manufacturing of final composite parts would help to omit a number of high-cost trial and error operations, the development of both experimental and theoretical approaches to analyze shear behavior of fibrous materials is of considerable interest.

The first approaches for the prediction of structural changes and mechanical characteristics of dry fibrous materials date back to the 1930s [1-3]. Since then, the conducted research has not obtained extensive results in comparison to other materials domains, though the necessity to handle complex fibrous materials properties has grown. The developments have been limited by the computer memory capacities. Now technical progress of today favors further theoretical research in this field. Besides, experimentally it has become possible to observe fibrous materials not only from its surface, but also inside with the help of the X-Ray tomography and full-field Digital Image Correlation (DIC) measurements [4]. This is of particular value, because the porous structure of fibrous materials transmits them the geometrical heterogeneity: their variety can be classified as it is presented in Fig. 1.

Another feature of these materials is their multi-scale architecture: microscopic constituents (fibers) are assembled into yarns, representing mesoscopic scale, which in turn create macroscopic pattern of the material. At 
Fig. 1 Structural classification of fibrous materials

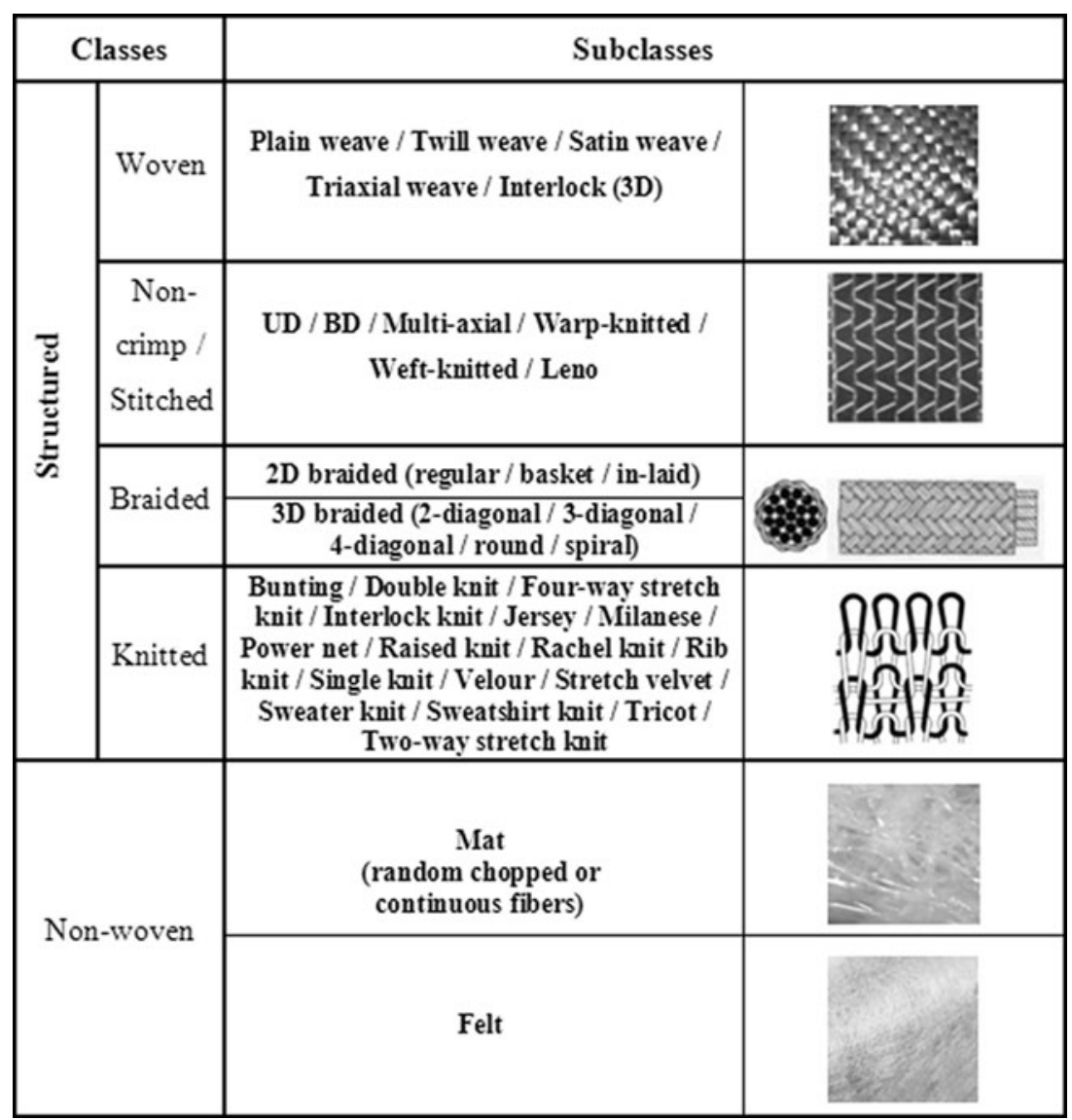

meso-scale, for periodic materials the smallest repeated pattern can be defined. It is called the Representative Unit Cell (RUC). The analogous concept exists for the random heterogeneous fibrous materials - the Representative Elementary Volume (REV). Though its definition is more complicated because it should fulfill the conditions for the size, which has to be large enough to own all possible properties and configurations of meso-constituents, and small enough not to possess a macroscopic heterogeneity anymore.

It should be noted that the tensile stiffness of fibrous materials in the yarns direction is several orders of magnitude higher than all the other rigidities. The bending and in-plane shear stiffness are low. The material is bent and draped easily, as opposed to the continuous materials such as polymer composites, or metals. In contrast to them, fibrous materials are characterized by the low cohesion between their constituents (between yarns at meso-scale, and between fibers at micro-scale) caused by the possible relative sliding between them and not perfectly straight structure. Even though the in-plane shear stiffness is low, it should not be neglected in modeling because during draping it is responsible for the wrinkles apparition, and for the woven and non-crimp materials the in-plane shear strains are the principal strains during draping $[5,6]$. With the gradual increase in shear angle, the lateral compression and crushing of yarns as well as changes in their cross-sections shape happen at meso-scale [7]. Therefore, the analysis at meso-scale should include the occurred local transverse compression field [8]. High compressibility is one more particularity of fibrous materials. The experimental analysis of fibrous materials shear behavior is often intricate, because meso-scale phenomena of yarns crushing, sliding with respect to each other and changes of the yarns crosssections are hard to capture with any kind of shear tests [7, 9-11]. The simple shear test on the fibrous material is not easy to perform either because simple shear implies the maintenance of two opposite specimen sides parallel to each other and with the constant length. Consequently, while being sheared, other two sides stretch out, and the specimen deforms into a parallelogram. Therefore, theoretical models for the prediction of the material response to the shear gain in high importance. Let us outline the reviews available in the literature on the models for mechanical properties of dry fibrous materials.

Since the numerical approaches for the prediction of fibrous materials behavior, as it was already mentioned, started to evolve with the technical progress, in 1980s, the first review of them was made by [12] with the 
description of challenges and trends for the future. The review was related more to the domain of computational fabric mechanics because the application of the considered models was the textile and garment industry, and rarely the composite materials industry. The main classification of numerical models divided them into continuum and discontinuum ones, mentioning separately geometrical and particle-based models. In the review paper [13] the approaches for modeling cloth, describing it at mesoscopic and macroscopic scales, were classified into geometrical, mechanical, and hybrid, which occupies the intermediate place between the first two types. Similarly, the emphasis was made on the models predicting the draping appearance properties and fabric hand, rather than mechanical properties, though particularly shear participates actively in draping deformations and defines wrinkles apparition. Hence later, [14] paid special attention to the shear prediction while considering the models for draping of woven and noncrimp materials. The authors in [14] described the essence and problems of geometrical and finite elements analysis (FEA) models, giving several examples of calculations by these models and their validation. Only macroscopic and mesoscopic models were under consideration. The review of FEA models for the prediction of woven fabric forming was made in [15]. They were classified into the continuous, discrete, and semi-discrete FEA. The latter was represented by the sole approach owing the features of both continuous and discrete approaches, and accounting for the tensile and shear properties. Continuous models were represented by the hypoelastic and non-orthogonal approaches. Later, in [16] the comparison of FEA models according to the same classification was performed. This time the results of hypoelastic, hyperelastic approaches (as the representatives of continuous FEA) were compared to the results of discrete and the improved semi-discrete approach that included simulation of tensile, shear, as well as bending properties. None of the review works have presented the real distinction of models according to the three scales of fibrous materials architecture. This is the guideline for this paper. The previous work of authors [17] has addressed such classification, having distinguished therewith the models for the prediction separately the bending properties and the tensile properties.

Therefore, the goal of this work is to provide an overview of the earlier studies made over the past decades to theoretically investigate particularly the shear properties and behavior of fibrous materials. The paper is organized according to the hierarchy of models with respect to the scale (Fig. 2): models addressing the behavior at the whole material parts scale; models taking into account yarns behavior at mesoscale; and models with the implemented behavior of constituents at fiber scale. In each section the approaches are also systematized relative to their mathematical formulation and framework.

\section{Macroscopic (whole component) scale}

The shear behavior of fibrous materials can be investigated with the following experimental techniques: picture frame shear, reproducing the pure shear mechanisms (Fig. 3a), bias extension (Fig. 3b), simple shear deformation (Fig. 3c), and forming to the hemisphere (Fig. 3d). The first three configurations are used for the shear characterization, while the hemispherical forming serves for the validation of the theoretically predicted deformations, and for the shear deformation, inherent in forming, in particular.

As it was already mentioned, draping is realized mainly by the in-plane shear mode particularly for the woven and non-crimp (NCF) fabrics. The models for these two classes of fibrous materials are thus considered in the sequel. The shear behavior of NCF differs from the one of woven fabrics due to stitching, binding two plies of angle-oriented tows, and causing the dependence of the behavior on the direction of deformation. Therefore, the shear stiffness of NCF is often referred to as "apparent shear stiffness" being superimposed on the tensile stiffness of the stitch. Thus the corresponding subsections in the paper will help to evaluate the differences between the models for woven and for non-crimp materials.

\section{Woven materials}

\section{Geometrical approach}

The advantage of applying the macroscopic methods to predict the material shear response is that they do not necessitate the preliminary measurement of small-scale parameters of the material to be input (like, for example, the width of yarns, yarns cross-sectional area - see Section "Mesoscopic (yarn) scale" and "Microscopic (fiber) scale"). The first models [22-24] were able to address only a macroscopic scale of the material as they were purely kinematic, i.e. independent of the material construction (yarn spacing, yarn size, weaving) and local mechanistic effects such as friction between yarns, or yarn compaction. These models represent a geometrical, the so-called pin-joint (or mapping) approach. They depict the material as a combination of points and lines of zero width (thus in the literature they are also referred to as "fishnet" approach) and usually deal with a single layer (Fig. 4).

The works by Potter [22] and Robertson et al. [23] investigated the rearrangement of the sheared yarns particularly during forming of a flat woven fabric to a sphere. Potter included the effect of yarns extension into his model, while Robertson et al. assumed the fabric components to be inextensible, considering these effects, often caused by the presence of yarn undulations, to be negligible in comparison to the overall deformation. Another assumption typical for 
Fig. 2 Classification of models for the prediction of shear response of fibrous materials

\begin{tabular}{|c|c|c|}
\hline Scale & Theoretical model & References \\
\hline \multirow{3}{*}{$\begin{array}{c}\text { Whole } \\
\text { component } \\
\text { scale }\end{array}$} & Geometrical approach & $\begin{array}{l}\text { [Bergsma and Huisman, 1988], [Long, 2001] (extended), } \\
\text { [Robertson et al, 1981], [Potter, 1980] }\end{array}$ \\
\hline & FEA Continuous & $\begin{array}{l}\text { [Aimene et al,2008],[Aimene et al,2010],[Khan et al,2010], } \\
\text { [Peng and Cao, 2005], [Thije et al, 2007], [Yu et al, 2002], } \\
\text { [Yu et al, 2005], [Xue et al, 2003], [Badel et al, 2009], } \\
\text { [Charmetant et al, 2012] }\end{array}$ \\
\hline & $\begin{array}{l}\text { Discrete energy } \\
\text { based approach }\end{array}$ & [Ben Boubaker et al, 2007] \\
\hline \multirow{7}{*}{ Yarn scale } & Geometrical approach & [Prodromou and Chen, 1997], [Rozant et al, 2000] \\
\hline & FEA Continuous & $\begin{array}{l}\text { [Badel et al, 2009], [Boisse et al, 2005], [King et al, 2005], } \\
\text { [Xue et al, 2005], [Badel et al, 2008], } \\
\text { [Page and Wang, 2002], [Lin et al, 2008] }\end{array}$ \\
\hline & FEA Discrete & [Creech and Pickett, 2006] \\
\hline & FEA Semi-discrete & $\begin{array}{l}\text { [Boisse et al, 2006], [Zouari et al, 2006], [Bel et al, 2012], } \\
\text { [Hamila and Boisse, 2007], [Hamila and Boisse, 2008] }\end{array}$ \\
\hline & $\begin{array}{l}\text { Continuous energy } \\
\text { based approach }\end{array}$ & $\begin{array}{l}\text { [Zhu et al, 2007], [Zhu et al, 2009], [Wiggers et al, 2003], } \\
\text { [Lomov et al, 2003], [Lomov and Verpoest, 2006] }\end{array}$ \\
\hline & Force based approach & $\begin{array}{l}\text { [Sun and Pan, 2005], [Page and Wang, 2000], } \\
\text { [Page and Wang, 2002] }\end{array}$ \\
\hline & Empirical approach & [Lomov and Verpoest, 2006] \\
\hline \multirow{2}{*}{ Fiber scale } & Geometrical approach & [Hofstee and van Keulen, 2001] (extended) \\
\hline & FEA Discrete & [Durville, 2007a], [Durville, 2007b], [Durville, 2010] \\
\hline
\end{tabular}

the pin-joint models states that the yarn crossings are fixed, with no slippage between yarns at these points that can occur in the reality. According to the approach by Robertson et al., the net representing a fabric is laid on the quadrant of the hemisphere starting from its top, a pole, and locating consequent nodes of the net by going from the right boundary of the quadrant downward, column by column, to the left boundary of the quadrant until the entire net (or the nodes of the net) is in contact with the hemispherical surface
(Fig. 5). The forming to the one quadrant only is performed reasoning from the symmetry stated by the authors, however, this symmetry can be experimentally confirmed only for the balanced woven materials [21, 26].

The position of each subsequent node is defined as the intersection of three spheres: two ones with the centers in adjacent nodes with known positions and of radii $a$, and the main hemisphere. Hence for the arbitrary node $(m, n)$, at location $\left(X_{m, n}, Y_{m, n}, Z_{m, n}\right)$, the following set of

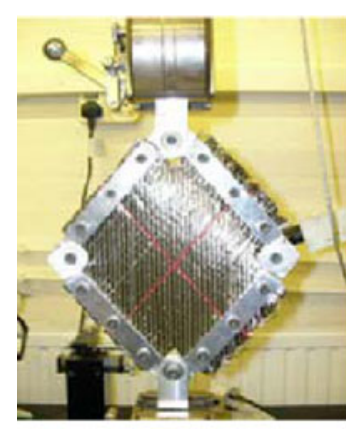

a)

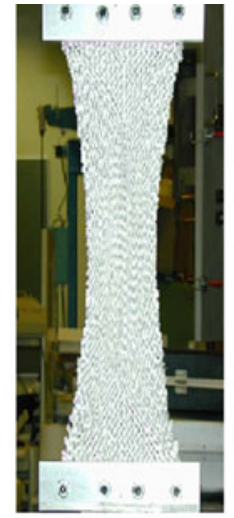

b)

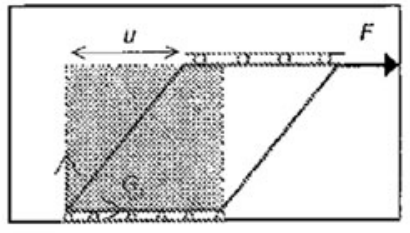

c)

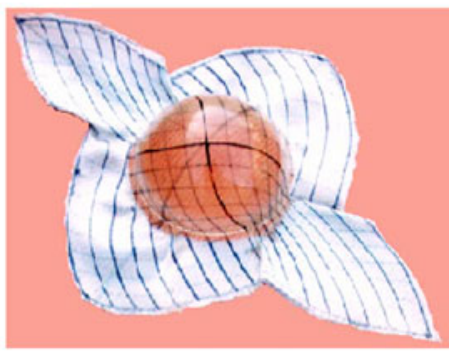

d)

Fig. 3 a Picture frame (pure) shear [18]; b shear deformation in bias extension [19]; c simple shear [20]; d hemispherical forming [21] 
Fig. 4 Schematic representation of shear deformation of woven fabrics [25]

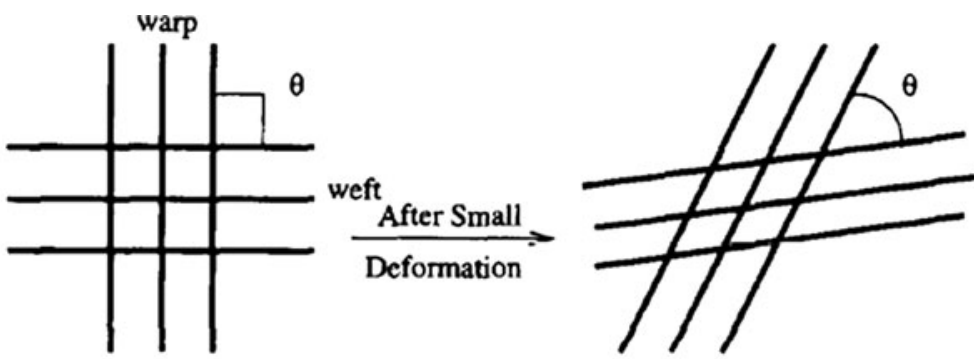

Initial State

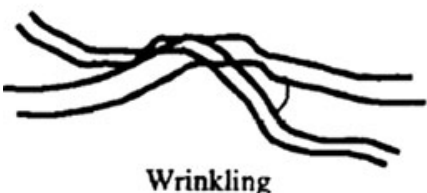

Deformation

Beyond Locking Angle

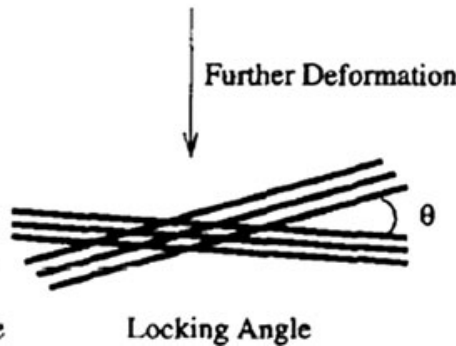

equations developed initially by Sickafus and Mackie [27] for the packing of spheres was solved:

$$
\left\{\begin{aligned}
&\left(X_{m, n}-X_{m-1, n}\right)^{2}+\left(Y_{m, n}-Y_{m-1, n}\right)^{2} \\
&+\left(Z_{m, n}-Z_{m-1, n}\right)^{2}=a^{2} \\
&\left(X_{m, n}-X_{m, n-1}\right)^{2}+\left(Y_{m, n}-Y_{m, n-1}\right)^{2} \\
&+\left(Z_{m, n}-Z_{m, n-1}\right)^{2}=a^{2} \\
& X_{m, n}^{2}+Y_{m, n}^{2}+\left(Z_{m, n}-R\right)^{2}=R^{2}
\end{aligned}\right.
$$

where $R$ is the radius of the main hemisphere, $a=$ $2 R \sin (\pi / 4 M), M$ is the number of nodes along the boundary of the quadrant including the equator and excluding the pole. The intersection the furthest from the pole is chosen among the solutions of equations. The proposed technique aimed at predicting a shear angle in yarn rearrangements, investigating the uniqueness of these possible rearrangements, and, in the case of non-uniqueness, analyzing the occurrence of wrinkles. It was found for the quadrant, and thus for the upper hemisphere, that no wrinkle appeared - a conclusion made out of the found symmetry about the diagonal conditions: $X_{m, m}=Y_{m, m}, X_{m, n}=Y_{n, m}$, $Z_{m, n}=Z_{n, m}$. The obtained yarn rearrangements with shear

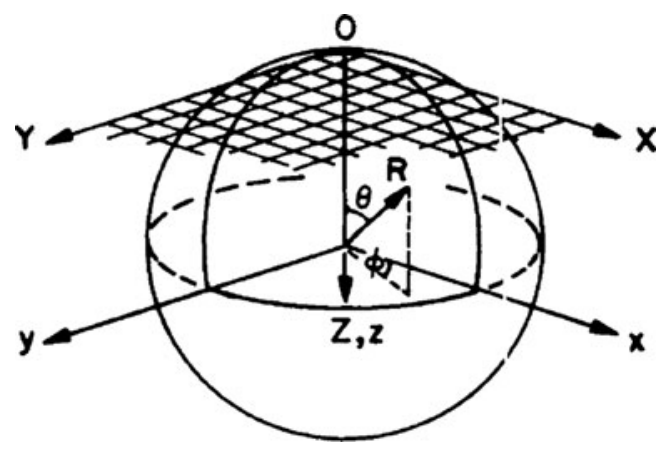

Fig. 5 Laying of the fabric "net" on a quadrant surface of the hemisphere [23] angles were compared to the experimental observations of the cheesecloth draped over a sphere, and showed a good fit. Besides, the extrapolation of the curve for the shear angle along the upper hemisphere gives the same occurrence of wrinkling in the lower hemisphere at a vertical distance of $1.7 R$ from the upper pole as in the experiments.

In pin-joint models the material deformation pattern is highly dependent on the choice of starting point and yarn directions. For many geometries this position is obvious, for instance, the highest point of a hemisphere. But more complex geometries may have multiple initial contact points, which complicates the simulation of the forming process, for instance, forming with a punch and a die.

The mapping approach was improved by Long [26], who extended the capacities of the approach and applied it to the woven satin materials. An iterative shear strain energy minimization scheme was used to find a deformed shape of the net. This improvement has distinguished more or less this technique from the class of purely geometrical ones. The total energy was calculated by summing the contribution at each node of the net (i.e. yarn crossover) $U_{s}$ :

$U_{s}(\vartheta)=\int_{0}^{\vartheta} T(\gamma) d \gamma$,

where $T(\gamma)$ is the torque required to reach a shear angle $\gamma$. The data for the shear energy necessary to be input in the scheme was obtained from the picture frame test (Fig. 3a). Modeling of draping of 4-harness satin weave over a hemisphere gave accurate prediction of shear angles.

\section{FEA continuous}

A number of macroscopic models were developed to be used in continuous finite element formulation [19, 28-30] taking into account, at the same time, implicitly the yarn 
directions from the mesoscopic scale. The difference in their formulations requires different parameters to be input, and correspondingly makes a model to be attached to one or another experimental test, which is sometimes not advantageous. The models also differ by the mechanisms during the shear deformation taken into account, for instance, accounting or not for friction effects. There are some models focused on a particular stage of shear deformation: whether it is a prediction of the initial shear displacements, or the shear force-angle relation is predicted up to the onset of wrinkles.

Xue et al. [28] proposed the non-orthogonal constitutive model for large deformations, where the stress-strain relationship in the global coordinates is defined by linking the analysis in the global orthogonal coordinate system and the non-orthogonal material coordinate system (Fig. 6a), and using the rigid body rotation matrices.

Assuming the tensile and shear responses of the material to be decoupled, the adequacy of which had been proved in the experiments earlier, the authors formulated the constitutive equation in the local coordinate system $\left(O^{\prime}, X^{\prime}, Y^{\prime}\right)$ as $[\sigma]_{x^{\prime} y^{\prime}}=\bar{D}[\varepsilon]_{x^{\prime} y^{\prime}}$ with the material matrix $\bar{D}=T_{2} D T_{3}$ in $\left(O^{\prime}, X^{\prime}, Y^{\prime}\right)$, where $T_{2}$ and $T_{3}$ are $3 \times 4$ transformation matrices from non-orthogonal coordinates $\left(O^{\prime}, \xi, \eta\right)$ to the orthogonal $\left(O^{\prime}, X^{\prime}, Y^{\prime}\right)$, for stresses and strains respectively. $T_{2}$ and $T_{3}$ are defined in terms of angle $\vartheta$ (Fig. 6a). The material matrix $D$ in $\left(O^{\prime}, \xi, \eta\right)$ for balanced fabric, and the stress-strain relationship in the global coordinate system $(O, X, Y)$ read:

$\mathbf{D}=\left[\begin{array}{cccc}D_{11} & D_{12} & 0 & 0 \\ D_{12} & D_{11} & 0 & 0 \\ 0 & 0 & D_{33} & 0 \\ 0 & 0 & 0 & D_{33}\end{array}\right] ; \quad\left[\begin{array}{c}\sigma_{x} \\ \sigma_{y} \\ \tau_{x y}\end{array}\right]=R \bar{D} R^{T}\left[\begin{array}{c}\varepsilon_{x} \\ \varepsilon_{y} \\ \gamma_{x y}\end{array}\right]$,

where $R$ is the unit transformation matrix between the local coordinates $\left(O^{\prime}, X^{\prime}, Y^{\prime}\right)$ and the global ones $(O, X, Y)$ expressed in terms of angle $\alpha$ (Fig. 6a). The material matrix D was found by matching the calculated force-displacement curves with the available experiments of unequal biaxial tension and picture frame test. The shear angle, defined as the angle change from a right angle after shear deformation, was calculated as follows $\gamma=\frac{\pi}{2}-2 \cos ^{-1}\left(\frac{u}{2 a}+\frac{\sqrt{2}}{2}\right)$, where $u$ is the end nodal displacement along the $x$ direction and $a$ is the width of the square sample contained between the clamping bars of the frame. The validation of the shear behavior prediction was performed on the picture frame shear deformation with misalignment of yarns relatively to the frame edges, characterized by the so-called off-angle $\varphi$, defined as $\varphi=\varphi_{0}(1-2 \gamma / \pi)\left(\varphi_{0}\right.$ is the initial off-angle). The increasing off-angle showed the rapid increase in the force (Fig. 6b), as the misalignment causes the deformation to be dominated by tension, not by shear. The reasonable agreement with experiments was found, with some discrepancies attributed by the authors to the deficiency of angle measurements, or to the assumption of no-slip boundary conditions under clamps.

Peng and Cao in [19] questioned the physical meaning of the stress transformation and stress definition in the non-orthogonal coordinate system in [28]. They proposed to integrate a convected coordinate system, whose in-plane axes take directions of warp and weft yarns of woven fabrics, into continuous finite elements. The global non-orthogonal constitutive relation, describing large deformations, was derived by transformations between contravariant stress/covariant strain components, introduced in the convected coordinate system, and the Cartesian stress/strain components. The equivalent contravariant elastic matrix was determined by empirical testing of the loaddisplacement curves of the uniaxial tensile and bias extension tests. It was expressed through the Green-Lagrange strains, shown to give adequate tensile and shear trends, as opposed to covariant or logarithmic strains. The constitutive equation together with the developed fiber orientation

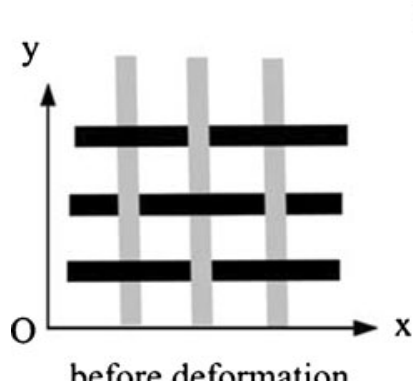

before deformation

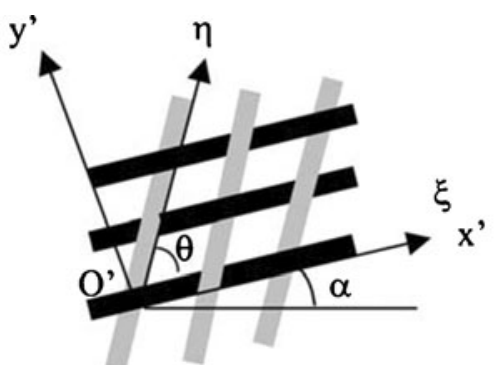

after deformation

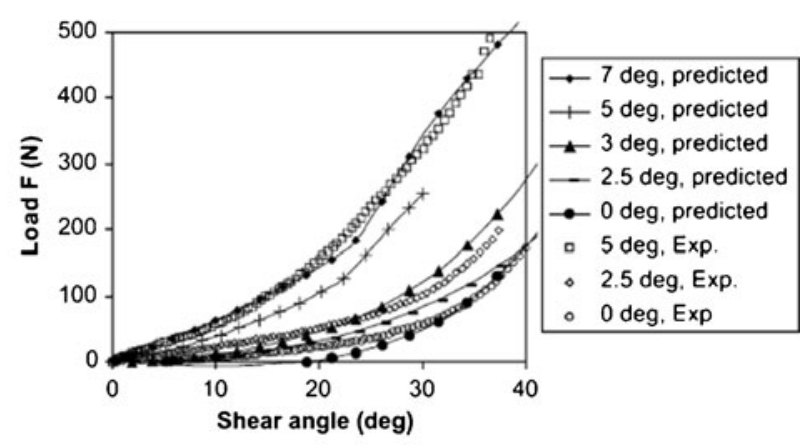

b)

Fig. 6 a Global, local and material coordinate systems for the fabric; $\mathbf{b}$ comparison of load vs. displacement curves for the predicted and experimental picture frame shear with misaligned reinforcements [28] 
model, based on fundamental continuum mechanics concepts, gave the results of the bias extension and picture frame shear simulations that agreed well with experiments (Figs. 7 and 8).

The bias extension modeling revealed the presence of three deformation zones (Fig. 7a): Zone I with the unchanged shear angle; Zone II with the combined shear and tensile deformations, and some change in angle between yarns; Zone III with significant shear deformation. Figure $7 \mathrm{~b}$ shows the contribution of the tensile deformation (in one of two directions): its highest values are located from the corners of the clamped end to the center of the free ends.

The thorough analysis of the picture frame shear was performed as well: setting material properties extracted from different shear frame tests, setting different misalignment angles between yarns and frame edges. The same influence of this angle on the shear behavior, as the one found in [28], was observed (Fig. 8).

Yu W. et al. [29] also proposed the non-orthogonal constitutive model for woven fabrics in the framework of the continuum FE approach, in which the material properties are determined in analytical form, explicitly relating stresses and strains as follows:

$$
\begin{aligned}
{\left[\begin{array}{c}
\Delta \sigma_{x x} \\
\Delta \sigma_{y y} \\
\Delta \sigma_{x y}
\end{array}\right]=} & {\left[\begin{array}{ccc}
\frac{\tilde{E}^{\alpha}}{b c}+\Gamma\left(\frac{a}{h}\right)\left(\frac{a^{2}}{c}\right) & \Gamma\left(\frac{a}{h}\right)\left(\frac{b^{2}}{c}\right) & \Gamma\left(\frac{a}{h}\right)\left(\frac{a b}{c}\right) \\
\Gamma\left(\frac{b}{\bar{a}}\right)\left(\frac{a^{2}}{c}\right) & \Gamma\left(\frac{b}{\bar{a}}\right)\left(\frac{b^{2}}{c}\right)+\frac{\tilde{E}^{\beta}}{\bar{a} c} & \Gamma\left(\frac{b}{\bar{a}}\right)\left(\frac{a b}{c}\right) \\
\Gamma\left(\frac{b}{h}\right)\left(\frac{a^{2}}{c}\right) & \Gamma\left(\frac{b}{h}\right)\left(\frac{b^{2}}{c}\right) & \Gamma\left(\frac{b}{h}\right)\left(\frac{a b}{c}\right)
\end{array}\right] } \\
& \times\left[\begin{array}{c}
\Delta \varepsilon_{x x} \\
\Delta \varepsilon_{y y} \\
2 \Delta \varepsilon_{x y}
\end{array}\right]
\end{aligned}
$$

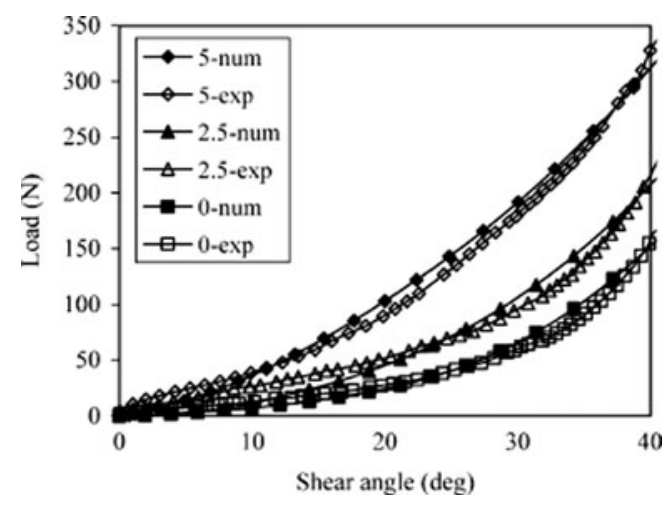

Fig. 8 Load-displacement curves in picture frame shear with yarns misalignments [19]

Here $\tilde{E}^{\alpha}=E^{\alpha} A^{\alpha}, \Gamma=A^{\gamma} E^{\gamma} /\left(a^{2}+b^{2}\right)^{3 / 2}$, where $A^{\alpha}, A^{\gamma}$ and $E^{\alpha}, E^{\gamma}$ are the cross-sectional area and elastic modulus of yarn respectively in $\alpha$ and $\gamma$ yarn directions (Fig. 9); $(a, b)$ is the material element of the $\gamma$ yarn based on materially embedded coordinate system; $\bar{a}$ and $\bar{b}$ are vectors showing the magnitude and direction of the $\alpha$ and $\gamma$ yarns in the unit cell (Fig. 9); $c$ is the thickness of the material.

The stress-strain relationships were derived under the assumption of small deformation using the kinematics and the force equilibrium of the related structural net (Fig. 9). Unlike the non-orthogonal models in $[19,28]$, the work by $\mathrm{Yu}$ W. et al. additionally accounts for the friction effect at the intersection points of fabric by means of elastic springs introduced between the diagonal intersection points in the

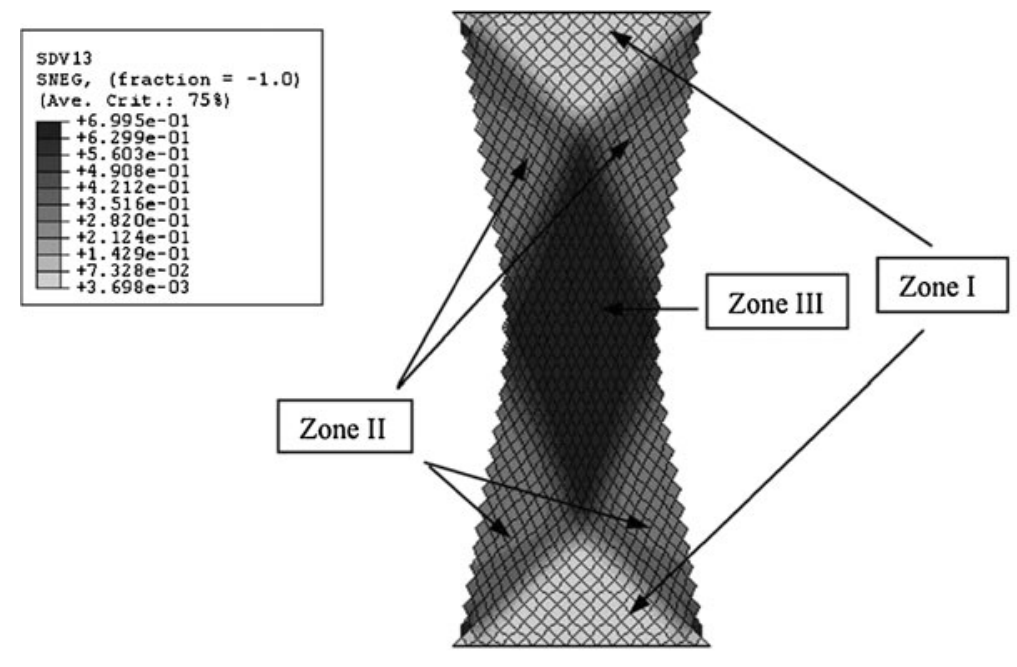

a)

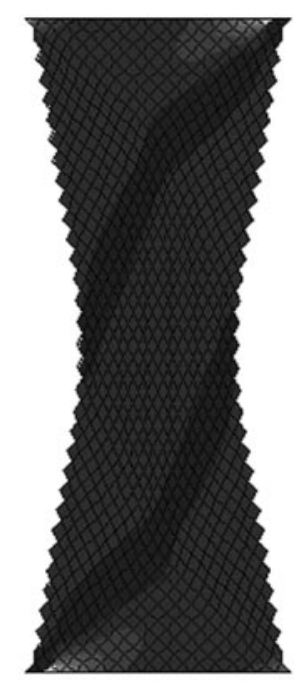

b)

Fig. 7 a Zones of shear strain in bias extension simulation; $\mathbf{b}$ contour of Green-Lagrange direct strain (responsible for stretching) in the $x$ weft direction in bias extension simulation [19] 
Fig. 9 A structural net (left) and a unit cell (right) of woven fabric in materially embedded coordinate system [29]

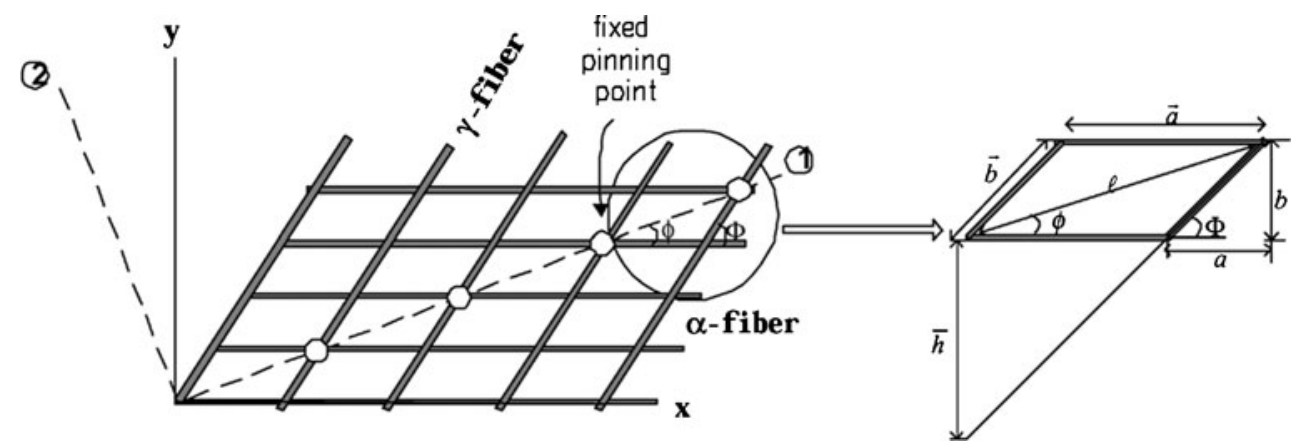

unit cell, and expressed as a resistance parameter $\tilde{E}^{\beta}$. The value of $\tilde{E}^{\beta}$ is chosen by fitting the experimental measurements, particularly, by fitting the fiber angle for the central point of the specimen in the bias extension test. The simulations of the bias extension up to $25 \%$ showed three typical distinct deformation regions described above, and good agreement with experiments. In simple shear and pure shear predictions the fact of not significant change in length of boundaries, compared in [29] to the predicted by the orthotropic constitutive law, confirmed the adequacy of the proposed non-orthogonal model. The simulation of the shear deformation of fabric formed over the hemispherical mold with a flat base (Fig. 10a) showed good agreement with experiments as well - for the angle values of maximal deformation in the equator region, $90^{\circ}$ angle values around the apex, and for the boundary profiles (Fig. 10b). A slight disagreement in the profiles in the weft direction was explained by the assumed equal properties for both warp and weft directions.

Recently, a hyperelastic objective constitutive model for large deformations of woven materials has been proposed by Aimene et al. [20,30] to be implemented in the continuum FE formulation. The hyperelastic approach is a good solution to address at macro-scale the strong nonlinearities of the material originating from its meso- and microstructural rotations. The potential is proposed in the form of the sum of tensile energies in two yarn directions, assumed to be uncoupled, and a shear energy, independent from the tensile one, each of which is a function of the right Cauchy Green and structural tensor invariants: $\mathrm{W}=\mathrm{W}_{1}\left(\mathrm{I}_{1}\right)+\mathrm{W}_{2}\left(\mathrm{I}_{2}\right)+\mathrm{W}_{C}\left(\mathrm{I}_{12}\right)$ with invariants $\mathrm{I}_{i}=\operatorname{Tr}\left(\underline{\underline{\mathrm{C}}} \cdot \underline{\mathrm{L}}_{i i}\right)=\lambda_{i}^{2}, i=1,2, \mathrm{I}_{12}=$ $\frac{1}{\mathrm{I}_{1} \mathrm{I}_{2}} \operatorname{Tr}\left(\underline{\underline{\mathrm{C}}} \cdot \underline{\underline{\mathrm{L}}}_{11} \cdot \underline{\underline{\mathrm{C}}} \cdot \underline{\underline{\mathrm{L}}}_{22}\right)=\cos ^{2} \vartheta$, where $\lambda_{i}$ are the yarn elongations, $\vartheta$ is the angle between yarns, $\underline{C}$ is the Cauchy Green strain tensor, $\underline{\underline{L}}_{i i}$ is the structural tensor, accounting for the material anisotropy: $\underline{\mathrm{L}}_{i i}=\underline{\mathrm{L}}_{i} \otimes \underline{\mathrm{L}}_{j}$ with $\underline{\mathrm{L}}_{i}$ - the initial yarn orientation. Then the second Piola Kirchhoff stress tensor $\underline{S}$ is expressed from the strain energy as: $\underline{\underline{S}}=2\left(\frac{\partial \bar{W}}{\partial I_{1}} \frac{\partial \overline{I_{1}}}{\partial \underline{\underline{C}}}+\frac{\partial \bar{W}}{\partial I_{2}} \frac{\partial \mathrm{I}_{2}}{\partial \underline{\underline{C}}}+\frac{\partial \bar{W}}{\partial \mathrm{I}_{12}} \frac{\partial \mathrm{I}_{12}}{\partial \underline{\underline{C}}}\right)$. Finally, it is derived that the components of the second Piola Kirchhoff tensor in the initial yarns orientation, divided by the Jacobian $J$ of the deformation, are equal to

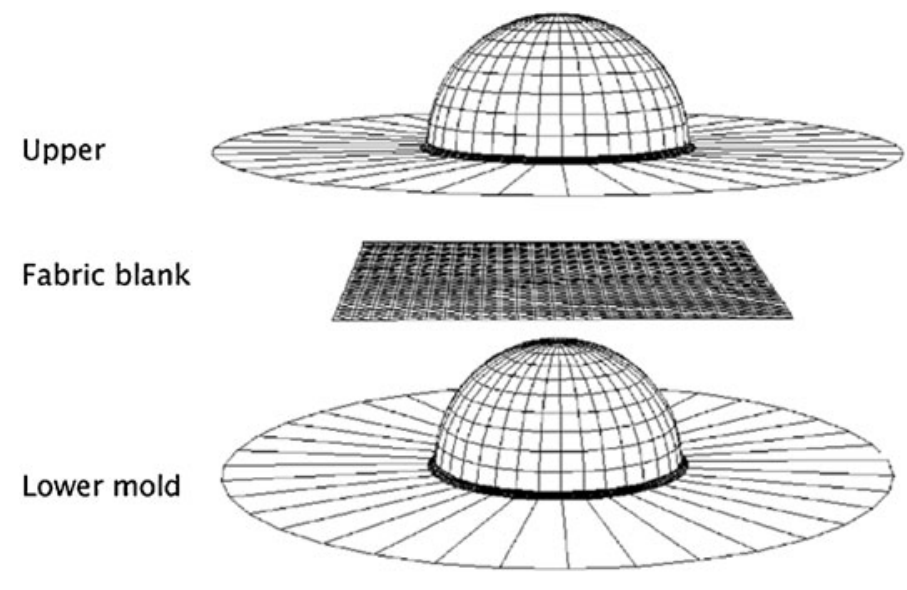

a)

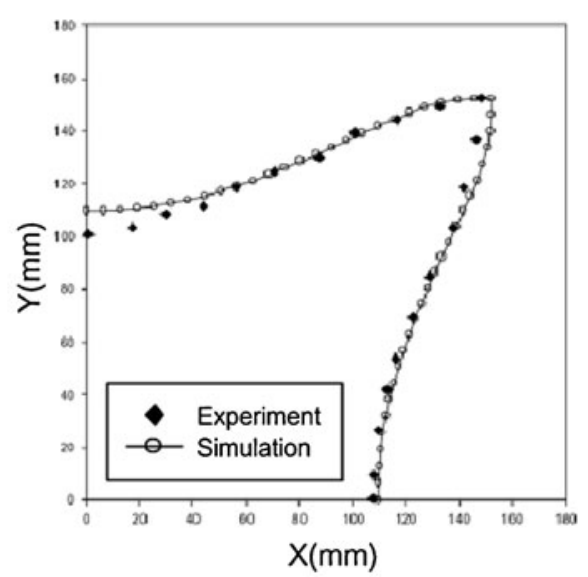

b)

Fig. 10 a FE model for the forming of woven fabric over a hemispherical mold; $\mathbf{b}$ comparison between experimental and predicted boundary profiles of draped fabric [29] 
Cauchy stress tensor components in the actual yarns orientation [20]: $\sigma^{i j}=\frac{1}{J} S_{i j}$. The potential is determined by fitting the data from two uniaxial tensile and a picture frame tests. The hemispherical forming simulations of plain-weave balanced and twill-weave unbalanced fabrics showed satisfactory qualitative correlation with experiments and importance of shear energy in describing wrinkle apparition. However, the results for balanced fabrics demonstrated better agreement with experiments in the case without accounting for shear energy, when there are no wrinkles. It was explained by the use of blankholder in experiments, helping to avoid wrinkles.

The same hyperelastic approach was applied by Charmetant et al. [31] to the prediction of large strains (including shear deformations) of 3D layer-to-layer angle interlock. Since this type of woven materials additionally owns the yarns through the thickness, perpendicular to other two yarns directions, its strain energy potential was built based on strain invariants representative to six deformation modes, compared to the three modes in [20]. Similarly to [20], the contribution of each deformation mode is assumed to be independent from the others, and thus allows additive composition of the strain energy density function. The deformation modes are expressed in tensile invariants in two yarn directions, transverse compression invariant, in-plane shear invariant, and two transverse shear invariants. The model parameters were identified from the following tests on fabric: tensile, compression, bias extension, and transverse shear (along warp and weft directions) realized by the special on-purpose device with two parallel planes fixed to the sample. The predicted by the hyperelastic model shear angles on top and bottom faces of draped over a hemisphere 3D interlock material [31] differ between each other and correspond well to the experimental data. Another advantage is the possibility to obtain predicted transverse compaction strains.

\section{Discrete energy based approach}

The discrete energy based approach developed by Ben Boubaker et al. [32] is also related to the macroscopic scale approaches because the woven fabric structure is represented by the two-dimensional lattice (trellis) with the nodes each owing a mass and a rotational rigidity (Fig. 11a), and the mesoscopic sizes (yarns width, spaces between yarns etc.) as well as the undulated geometry are not taken into account. At the same time, some underlying mesoscopic deformation mechanisms are included in the analysis: the nodes are connected by the bars that can exhibit extension; the unit cells, composed of set of four bars, are endowed with a torsion mode (Fig. 11b). The fabric is considered as an elastic structure, with the principal directions coinciding with the warp and weft directions. Thus the following mechanical parameters for the constituents of lattice need to be input in the model: the stiffness moduli in the warp and weft directions $E_{1}, E_{2}$, the shear modulus $G$, the contraction coefficients $v_{1}, v_{2}$ in the warp and weft directions. There is an equivalent elastic foundation represented by the extensional springs (Fig. 11a) located perpendicularly to the initial plane of the lattice. A relatively large variety of boundary conditions can be prescribed in the model due to the rotational rigidities given to the lattice edge nodes.

By minimizing the total potential energy the equilibrium shape of the structure is obtained. The strain energy of the lattice equals the sum of the bending, torsional, stretching and shearing energies (specified at each node), deduced from the work of the internal forces. The total strain energy of the discrete model is defined as a sum of the strain energy of the lattice, total stretching energy of the elastic foundation, and energy of the edge's supports. The work of the external forces is composed of terms of the work of the gravitational forces and the work of the applied mechanical loads. In the calculations, however, the authors assumed the kinematics to be dependent only on the local bending rotations, supposing the fabrics to be submitted to its own weight and to small loads. Therefore, the contributions of stretching and shearing energies were omitted. The problems of fabric draping over a square support, to the surface of which the fabric was attached, and draping over a hemisphere under the own weight were considered. These constrained problems, owing around 2500 degrees of freedom, were solved by the classical method of Lagrange multipliers. The stability analysis using the Dirichlet-Lagrange criterion was also performed in [32]. As a result, the critical buckling loads of the structure were found.

Evaluating the proposed approach from the point of view of practical realization, the inconvenience of large number of degrees of freedom should be mentioned. There is also a lack of estimation of accuracy of the obtained results in [32] (e.g. experimental comparison).

Non-crimp/stitched materials

\section{Geometrical approach}

All above mentioned macroscopic models are employed for woven fabrics. At the same time, growing industrial needs in non-crimp fabrics (Fig. 1) as composite reinforcements motivate the development of models to describe their deformation. And since the yarns - tows - of NCF are very stiff in tension, the fabrics deformation in the process of forming to the double-curved geometries is achieved mainly by the in-plane shear.

Suggesting in [33] to use the geometrical approach, Potter showed that it can adequately describe the global shear deformation of the non-crimp unidirectional crossplied prepreg subjected to bias extension, meaning the 


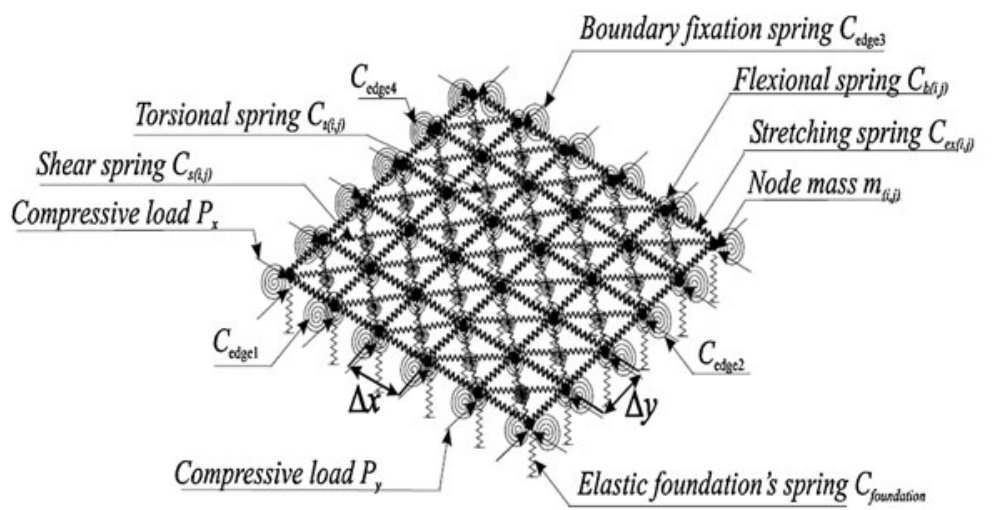

a)

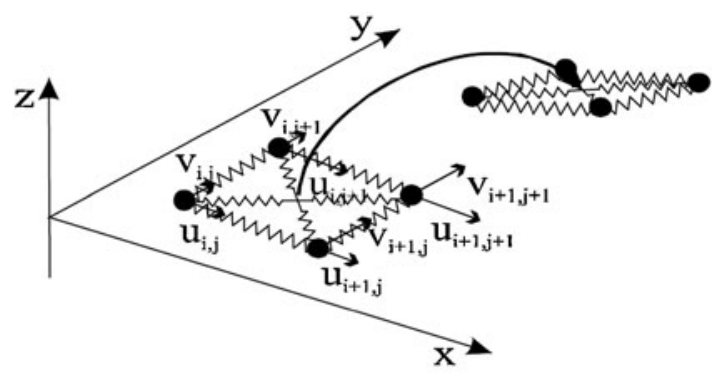

b)

Fig. 11 a Discrete model for fabric with fixed edges; b woven unit cell of fabrics with the illustrated shear deformation [32]

general relationships between the longitudinal and transverse strains. However, the observed in the experiment local deformation, like the local buckling of fibers (not affecting every tow in the prepreg), was different from the predicted one.

Improved geometrical (mapping) approach in [26] with the iterative strain energy minimization scheme, described in the previous section, was applied in [26] to predict the shear behavior of non-crimp materials as well. The model has proved to give accurate prediction of shear angles for a range of fabrics and geometries, such as $\pm 45^{\circ}$ pillar warp knit draped over a hemisphere; $\pm 45^{\circ}$ tricot 1 \& 1 warp knit formed over a jet engine nose-cone geometry.

\section{FEA continuous}

The non-orthogonal model in [29] was then extended by $\mathrm{Yu}$ W. et al. in [21] to predict shear deformations of noncrimp fabrics. To the constitutive Eq. (4) [29], accounting for fiber directional properties, eliminating the friction term with $\tilde{E}^{\beta}$, in [21] Yu W. et al. added the contribution from the non-linear shear properties of fabrics, defining the increment of stress $\Delta \sigma$ due to the increment of shear angle $\Delta \gamma$ as follows:

$\Delta \sigma=\left[\begin{array}{c}\Delta \sigma_{x x} \\ \Delta \sigma_{y y} \\ \Delta \sigma_{x y}\end{array}\right]=\left[\begin{array}{ccc}0 & 0 & 2 G_{1} g_{1}^{1} g_{2}^{1}+G_{2}\left(g_{1}^{1} g_{2}^{2}-g_{1}^{2} g_{2}^{1}\right) \\ 0 & 0 & 2 G_{1} g_{1}^{2} g_{2}^{2}+G_{2}\left(g_{1}^{1} g_{2}^{2}-g_{1}^{2} g_{2}^{1}\right) \\ 0 & 0 & G_{1}\left(g_{1}^{1} g_{2}^{2}-g_{2}^{1} g_{1}^{2}\right)\end{array}\right]\left[\begin{array}{c}0 \\ 0 \\ \Delta \gamma\end{array}\right]$,

where $G_{1}=\frac{1}{l c}\left\{\frac{d F_{s}}{d \gamma} \sqrt{g^{11}}+F_{s} \sqrt{g^{11}\left(g^{11}-1\right)}\right\}, G_{2}=$ $\frac{F_{s}}{l c} \sqrt{g^{11}}, F_{s}$ is the shear force, $g^{11}$ is the contravariant base vector, $l$ and $c$ are the side length and thickness of the fabric respectively, assumed to be constant in picture-frame shearing (the fact experimentally justified for dry NCF). After transforming Eq. (5) into the local coordinate system used in Eq. (4), which can change during shear deformation, Eq. (5) is then added to Eq. (4) to get the final constitutive equation. Shear force $F_{S}$ versus shear angle data were input into the constitutive equation from fitting the experimental picture-frame shear curves with exponential and polynomial functions for two regions. Being implemented then into the implicit FE code, the non-orthogonal constitutive model was reasonably validated against picture-frame test force-shear angle curves, demonstrating the asymmetric shear deformations of NCF (Fig. 12a). Some discrepancies were observed, and attributed by the authors to the difference in boundary conditions between the picture-frame test and the simulation.

The extensive hemispherical forming simulations with detailed parametrical studies on the influence of blankholder force, its shape, and friction effect were presented as well in [21]. It was found that both increasing the blankholder force, and increasing friction decreased asymmetry in shear angle distributions, usually observed for NCF forming even to symmetric geometries (Fig. 12b). The weakness of the obtained forming simulations is the disagreement with experiments in the equator region - the region with large deformations.

The solution to this problem of FE analysis of large deformations of highly anisotropic materials was proposed by ten Thije et al. in [34]. They decomposed the deformation gradient into a rotation tensor $\mathbf{R}$, reflecting the rotation of the axes of anisotropy, and a stretch tensor $\mathbf{G}$ as follows: $\mathbf{F}=\mathbf{R} \cdot \mathbf{G}$. Here the principle of the updated or total Lagrange FE formulation for anisotropic materials lies in the relation of the invariant (because it co-rotates with the rigid body rotations of the axes of anisotropy) local stress tensor $\tau$ to the global Cauchy stress tensor $\sigma$ by a rotation 


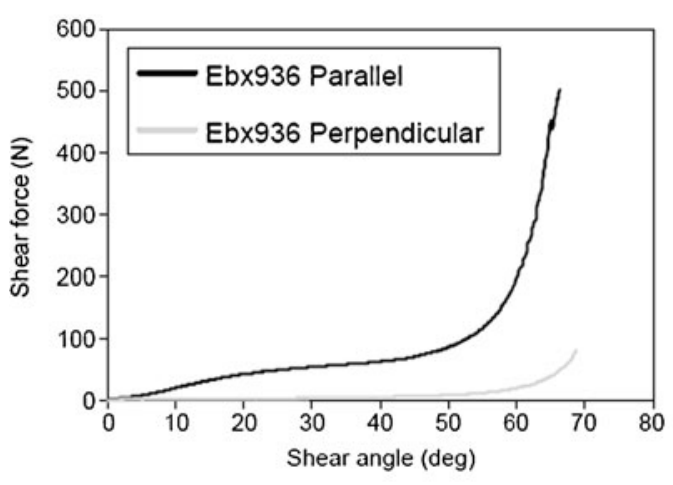

a)

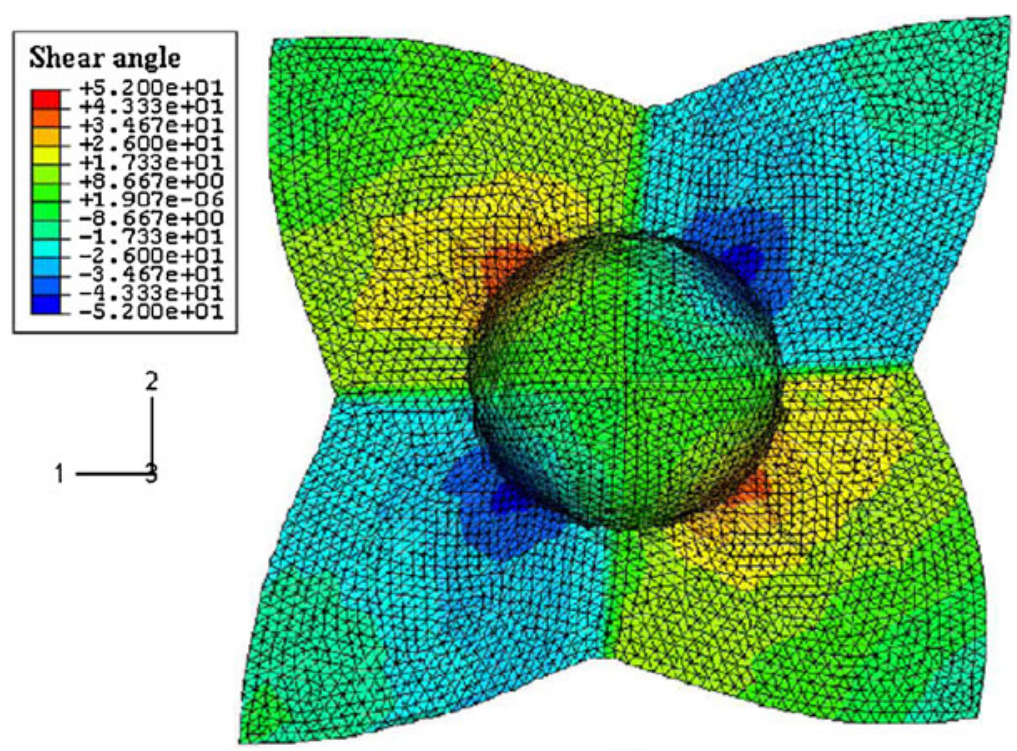

b)

Fig. 12 a Experimental shear compliance curves for the parallel and perpendicular to the stitch direction shear; $\mathbf{b}$ asymmetric formed shape of $\mathrm{NCF}$ [21]

only: $\sigma=\mathbf{R} \cdot \tau \cdot \mathbf{R}^{T}$. To address large deformations, the right $\mathbf{C}$ and left $\mathbf{B}$ Cauchy Green tensors were used and became as follows after decomposition of the deformation gradient:

$\mathbf{C}=\mathbf{G}^{T} \cdot \mathbf{G}, \quad \mathbf{B}=\mathbf{R} \cdot \mathbf{G} \cdot \mathbf{G}^{T} \cdot \mathbf{R}^{T}$.

Constitutive equations were derived using the Helmholtz free energy. The mechanical equilibrium without the presence of body forces, after the procedure of weighing, reduced integration and application of the divergence theorem of Gauss, in the rate form reads:

$\int_{V}\left(\mathbf{w} \overleftarrow{\nabla}: \dot{\sigma}-\mathbf{w} \overleftarrow{\nabla} \cdot \mathbf{v} \overleftarrow{\nabla}: \sigma+\mathbf{w} \overleftarrow{\nabla}: \sigma \frac{\dot{J}}{J}\right) d V$

where $J$ denotes the Jacobian, the volume ratio $J=$ $\operatorname{det}(\mathbf{F})=\frac{d V}{d V_{0}}, \mathbf{v}$ is the velocity, $\mathbf{w}$ are the weight functions. With the help of this equation, the consistent tangent matrix for the generalized elastic anisotropic material is then expressed as:

$$
\begin{gathered}
K=\int_{V}\left[\frac{\rho}{\rho_{0}} \cdot \frac{1}{2}(\mathbf{w} \overleftarrow{\nabla}+\vec{\nabla} \mathbf{w}):\left(\mathbf{F} \cdot{ }^{4} \mathbf{I} \cdot \mathbf{F}\right):{ }^{4} \mathbf{E}:\left(\mathbf{F}^{T} \cdot{ }^{4} \mathbf{I} \cdot \mathbf{F}^{T}\right)\right. \\
\left.: \frac{1}{2}(\mathbf{v} \overleftarrow{\nabla}+\vec{\nabla} \mathbf{v})+\vec{\nabla} \mathbf{w} \cdot \mathbf{v} \overleftarrow{\nabla}: \sigma\right] d V
\end{gathered}
$$

where ${ }^{4} \mathbf{I}$ is a fourth order tensor, ${ }^{4} \mathbf{E}$ is the invariant and constant fourth order material tensor.

In the bias extension simulation of NCF with two layers oriented at $\pm 45^{\circ}$ and the stitch at $0^{\circ}$, proposed by ten
Thije et al. [34], the stitch is considered to deform plastically and to dominate the shear deformation of the fabric. It is included in the formulation by additional fiber fraction deforming according to the Nadai stress-strain curve, with the initial linear region up to the yielding point and subsequent region described by: $\sigma_{y}=\sigma_{0}+C\left(\varepsilon_{0}+\varepsilon_{p}\right)^{n}$, where $\varepsilon_{p}$ is the plastic strain, $\sigma_{0}=30 \mathrm{MPa}, C=100 \mathrm{MPa}$, $\varepsilon_{0}=5 \times 10^{-5}$ and $n=0.6$ are taken as material parameters. The results of the bias extension simulations showed the distribution of the stitch plastic strain with $40 \%$ maximum value.

Thus the proposed FE formulation successfully copes with non-crimp materials large deformation simulations, moreover, demonstrating quadratic convergence of calculations. However, there is a lack of quantitative comparison of simulated shear deformations with experiments in the work.

\section{Mesoscopic (yarn) scale}

After the initial stages of shear deformation the material behavior starts to be greatly influenced by its architecture, i.e. meso-structure, like yarn width, yarn spacing, which are not taken into account in macro-scale models. It is also influenced by mechanistic effects, like friction (slippage) between yarns, as well as inter-ply interactions (for multilayered structures) and local reduction in global stiffness due to lay-up orientation [25]. The completeness of the mesoscopic model can thus be estimated by a quantity of the mentioned factors and mesoscopic mechanisms addressed 
in the model. Different approaches address different stages of the shear deformation. Some of mesoscopic approaches are devoted mostly to the late stages of shear deformation, i.e. when wrinkling (out-of-plane buckling) occurs after a certain in-plane shear angle value is reached (except for the shear behavior in bias extension test).

Woven materials

\section{Geometrical approach}

Prodromou and Chen proposed a model in [25] based on the pin-joint theory and simultaneously accounting for the aforementioned fabric geometrical parameters to predict the shearing behavior and locking angle apparition.

Reasoning from the geometry (Fig. 13), the shear angle versus percent displacement curve was obtained according to the expression:

$$
\begin{aligned}
\cos \vartheta & =\cos \vartheta_{0}\left(1+\frac{A D-A D_{0}}{A D_{0}}\right) \\
& =\cos \vartheta_{0}(1+\% \text { displacement })
\end{aligned}
$$

where $\vartheta_{0}$ is the initial angle, $\vartheta$ is the shearing angle, $A D$ denotes half the length of the unit cell in the bias direction, $A D_{0}$ denotes the value of $A D$ at $0 \%$ displacement. It showed the same trend as experimental data from the picture frame test performed in [25]. However, at final stages of the displacement the substantial scatter between the experimental and predicted values was observed.

In [25] it was proposed that the locking angle occurred at the point, where the distance between the warp and weft yarns was zero, because the locking angle is defined as the highest shearing angle corresponding to the observation of wrinkles. The formula expressing that locking angle is: $\vartheta=\arcsin (t / L)$, where $t$ is the tow width, and $L$ is the tow spacing (distance between the centers of successive tows). The comparison of measured and predicted locking angles showed deviations in the values. This illustrates the weakness of the pin-joint theory in describing the shear behavior. The possible reason for that is that the mechanistic effects such as friction between the yarns, variability of the tow width (which can be observed initially along the tow, or
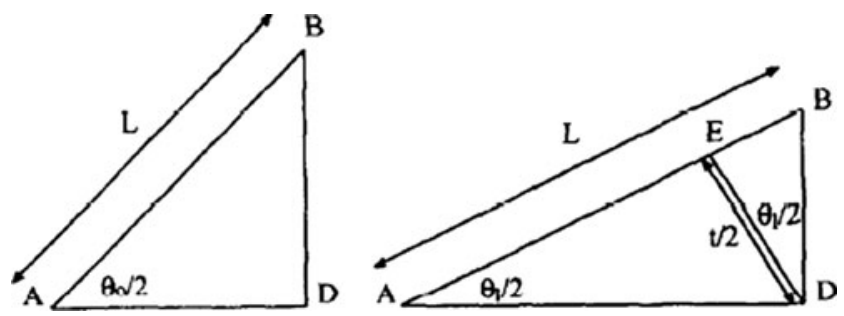

Fig. 13 One quadrant of the unit cell in pin-joint model: initial position and locked position [25] after some time during shearing), and/or tow spacing are not taken into account. The authors provided the evidence of the influence of these factors by analyzing friction in the pullout test on the fabrics, and performing a parametric study of the tow width and spacing variations. However, accounting for these effects in the model was only mentioned as a perspective.

Rozant et al. [35] investigated the drapeability of fabrics and predicted the locking angle in the same manner as Prodromou et al., but calculating it independently for the warp and weft yarn directions, which most often have different yarn sizes and spacing. Then the highest of the two obtained values was chosen as the locking angle.

\section{Force based approach}

The force based approach, working on the principle of equilibrium of forces within the deformed material, was used by several groups of authors [36-38]. The analysis of different shear configurations was performed: bias extension and pure shear. Different works also considered different stages of shear deformation, one of the major phenomena of which is the slippage at yarns intersections that is considered to be an indicator of the transition to the next shear deformation stage. The influence of different material parameters on the occurrence of slippage was studied as well.

In [36, 37] Page and Wang proposed an approach based on the forces equilibrium and beam deflection theory in order to include the above-mentioned mechanistic effects in the analysis of plain woven fabrics at meso-scale. Considering the uniform shear deformation, i.e. with the same shear angle everywhere in the pattern of fabric subjected to bias extension, three stages of the shear state were analyzed: initial shear without sliding owing to the friction at yarns intersections; shear with sliding at intersections; and larger shear deformation (but until the yarns contact side by side) accompanied by elastic resistance. In the first stage, characterized only by slight bending of yarns, based on the beam deflection theory, the deformation was calculated to be very small and, therefore, neglected. In the second stage with sliding, the force based approach gave the shear force $F_{u}$ expressed in terms of $\vartheta^{\prime}=\vartheta / 2(\vartheta$ was the angle between yarns) as:

$$
F_{u}=\frac{\sqrt{2} N_{b} u I_{\vartheta}}{L^{2} \sin \vartheta^{\prime} A-2 L \cos \vartheta^{\prime} \tan \alpha u I_{\vartheta}}
$$

where $\quad A=\frac{1}{2} w^{2}\left[\tan \vartheta^{\prime}+\tan \left(90^{\circ}-\vartheta^{\prime}\right)\right]$,

$$
\text { and } \begin{aligned}
I_{\vartheta}=\frac{1}{12} w^{3}\left[\frac{\sin \vartheta^{\prime}}{\cos ^{2} \vartheta^{\prime}}+\frac{\cos \vartheta^{\prime}}{\sin ^{2} \vartheta^{\prime}}\right. & +\ln \tan \left(45^{\circ}+\frac{\vartheta^{\prime}}{2}\right) \\
& \left.-\ln \tan \left(\frac{\vartheta^{\prime}}{2}\right)\right] .
\end{aligned}
$$


The normal force $N_{b}=$ const between yarns due to bending, inter-yarn friction coefficient $u$, yarn length $L$ in the RUC, weave angle $\alpha$, and yarn width $w$ were input from measurements and characterization tests. The third stage was proposed to be described by Eqs. (10), but with $N_{b}=N_{b}(\vartheta)$, prescribed as the evolution of the normal force with the increasing shear as a result of growing friction. $N_{b}$ was obtained from the FEM simulation of the fabric RUC with its internal forces owing to weaving [37]. Thus the non-linear shear force-angle prediction correlated well with the bias extension test data. Reasoning from the symmetrical deformation of every RUC in uniform shear, it was concluded that the slippage can occur when the uniform distribution of angles is violated, and it is the more probable the higher the bending rigidity of yarns is.

Later, Sun and Pan [38] also applied the force based approach to obtain the force-displacement relationship for the two initial stages of in-plane shear deformation (without and with sliding at yarns intersections) of plain woven fabrics. The case was considered as the pure shear because at small shear angles the tensile components of forces own negligible magnitude. The model required the following geometric and material properties of yarns to be input: the spacing between adjacent picks of yarns for warp and weft directions, the yarn thickness and length, the contact length of yarns, the yarn Young's modulus, the shear modulus of the yarn cross-section. The expressions for the yarns rotational angles were derived from the bending and torsional moments at any cross-section in the case of nonsliding. Considering the case of sliding at the contact zone, the shear force was obtained from the sliding equilibrium between the external moment due to the shear force and the frictional moment. Comparison of the obtained shear force/angle curves, as well as the limits of sliding regions (in degrees) with the experimental ones showed reasonable agreement. Satisfactory correlation was also observed between the predicted average shear modulus, calculated by the linear interpolation, and the experimental one, however, with the tendency of the model to stiffen the material response.

\section{Continuous energy based approach}

The proposed in the literature models based on the energy minimization principle [39-41] differ one from each other by different definition of the constituent energies composing the total energy. Consequently, the accuracy of results, obtained by different models, can be evaluated according to the included in the analysis energy terms that correspond to the mesoscopic mechanisms induced by the shear deformation, e.g. the slippage at yarns cross-overs, the yarns transverse compression, the yarns rotation, and according to the way the mechanisms are decoupled in the model.
The mechanistic effect of yarns slippage during the shear deformation in three experimentally identified stages of the bias extension was investigated by Zhu et al. [40]. In each of the stages the total energy consisted of several of the following components: in-plane shear, yarn tension and/or inter-yarn slippage contribution. The first stage is defined as the one during which the plain woven fabric owns seven regions (or three symmetrical to each other zones - see Sect. 1) with different shear angles; besides there is increasing tension along yarns. Thus the total energy contains the shear component, obtained in the picture frame test, and the tension component, which was assumed in this stage to increase linearly from zero to the value of the slippage energy that occurs in the second stage. The distinct separation of three shear zones is violated in the second stage because the yarns cannot be further tensioned, and the slippage of yarns starts to expand from the middle of the loaded material area to the top and bottom, as observed in the tests. Therefore, the slippage energy should be added to the total energy, and is expressed in terms of lateral compressive force between yarns [40]. The magnitude of the force was obtained in a lateral compression test. In the third stage the shear energy remains constant because there is no further shear deformation. The slippage energy is identified similarly, but only in the bottom region of the specimen, where the slippage is localized during this stage.

The method is convenient for the comparative observation of each mechanism contribution to the bias extension behavior because it is possible to estimate them decoupled: the separate graphs of the shear, tension, and slippage energies versus displacement were presented. The predicted total energy was a little lower than the experimental one, particularly in later stages. It was explained by the linear assumptions, by the yarn bending along the boundaries of different regions, and by the non-uniformity of the distribution of zones of identical shear angle. The inconvenience of the model is that most of the required input data have to be obtained from several different experiments.

Later, the same group of authors Zhu et al. [41] employed the energy based approach to predict the picture frame shear of plain woven fabrics up to large deformations and wrinkles apparition. The total in-plane shear energy was considered as a sum of the energy, dissipated by the rotational friction between yarns cross-overs, the sliding friction energy relating to the boundaries of the cross-over regions, and the transverse compression energy. The rotational friction energy was estimated following Kawabata et al. [42]. The two other components were expressed in terms of transverse compressive force to a yarn segment, identified from the lateral compression test of yarns. The results showed significant role of the yarn lateral compression at later shear stages, and negligible contribution of the rotational friction energy during all stages. The shear locking (wrinkling) 
criterion was proposed in the form of equality between the in-plane shear energy and the out-of-plane wrinkling energy. The latter was defined as the localized bending at the boundaries between four parts of the woven RUC (Fig. 14), with the neglect of change in the yarn geometry inside each of four parts. The predicted locking angle value and the load-displacement curve showed good agreement with experiments.

The continuous energy based model was also developed by Lomov et al. in [39] to predict the shear behavior of woven fabrics. The model calculated the sheared state of the fabric RUC, as a function of the shear angle, in the presence of (pre)tension, as an equilibrium between the internal energy change and mechanical work $A$ of the applied loads as follows: $M=2 T X Y \cos \gamma, A=\frac{1}{2} M \gamma$, where $T$ is the shear force; $\gamma$ is the shear angle; $M$ is a moment, $X$ and $Y$ are sizes of the RUC. The shear deformation is coupled to tensile effects, where yarns tensions (input from the test) and associated (calculated) transversal forces were assumed to be constant during shear. The mechanical work was composed of yarns (un)bending, torsion, friction, and vertical displacement. The yarns lateral compression was addressed by an additional component, defined from the experimental compression diagram of the yarn, in transversal forces calculation. The latter took part in the definition of the work of vertical displacement. For the other work terms the following parameters were required to be input from the tests: the friction coefficient and the bending rigidity, measured on KES-F (Kawabata Evaluation System for Fabrics). The torsional rigidity of the yarn $C$ was proposed to be estimated in terms of bending rigidity $B$ and the yarn thickness $d_{1}$ as follows: $C=\frac{B}{d_{1} / 2}$. Consequently, the proposed model allowed to obtain shear load-displacement curves, and to estimate the contribution of each mechanism to the shear resistance. At shear angle $45^{\circ}$ more than half of the work is spent on friction, then the smaller contribution is made by torsion and vertical displacement, the smallest - by bending, which

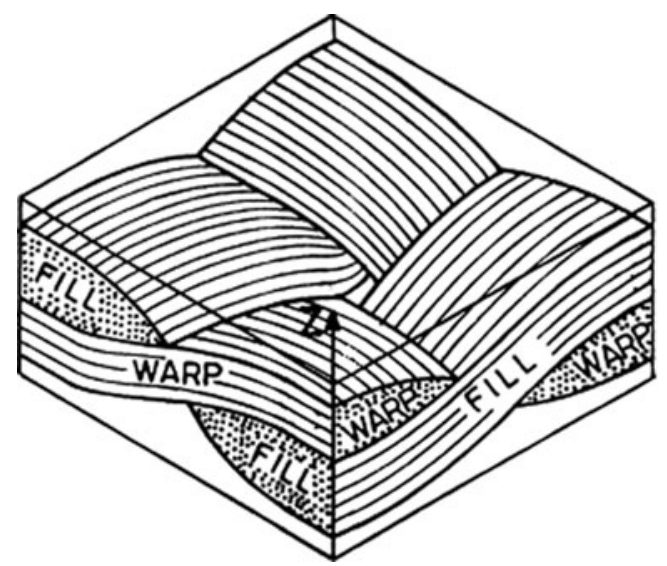

Fig. 14 Representative unit cell of plain-weave woven fabrics [43] decreases even more at a shear angle of $60^{\circ}$. On the contrary, the impact of friction and vertical displacement increases with the angle; the torsion gets weaker.

The built shear curves were further used by the same group of authors for the development of another type of model, whose principles are described in the next section.

\section{Empirical approach}

Following the work [39], Lomov and Verpoest [44] proposed then a phenomenological model that can be employed for the prediction of shear resistance of woven fabrics of different structure, with components having clear mechanical meaning. The suggested empirical relation had the form: $T(\gamma)=T_{0}+T_{1} \tan ^{\alpha} \gamma$, where $T_{0}$ defined the initial finite shear resistance, caused by the inter-yarn friction, before any occurrence of the displacement; $T_{1}$ defined the shear modulus. For the purpose of finding parameters $T_{0}, T_{1}$ and $\alpha$ by fitting, the shear curves from [39] were used. The parameters were identified by the regression analysis, and expressed in terms of fabrics lineal density $t$, looseness factor $s=\left(p-d_{1}\right) / d_{1}$ ( $p$ is the spacing of yarns) and pretension $\varepsilon$ as follows:

$$
\begin{aligned}
\ln T_{0}= & b_{1}+b_{2} \cdot \ln t+b_{3} \cdot \ln s+b_{4} \cdot \ln \varepsilon \\
& +b_{5} \cdot(\ln t)^{2}+b_{6} \cdot(\ln t \cdot \ln s) \\
& +b_{7} \cdot(\ln t \cdot \ln \varepsilon)+b_{8} \cdot(\ln s)^{2} \\
& +b_{9} \cdot(\ln s \cdot \ln \varepsilon)+b_{10} \cdot(\ln \varepsilon)^{2}, \\
\ln T_{1}= & c_{1}+c_{2} \cdot \ln t+c_{3} \cdot \ln s+c_{4} \cdot \varepsilon \\
& +c_{5} \cdot(\ln t)^{2}+c_{6} \cdot(\ln t \cdot \ln s) \\
& +c_{7} \cdot(\ln t \cdot \varepsilon)+c_{8} \cdot(\ln s)^{2}+c_{9} \cdot(\ln s \cdot \varepsilon) \\
& +c_{10} \cdot \varepsilon^{2} .
\end{aligned}
$$

After fitting, the parameter $\alpha$ showed its independence of $t, s$ and $\varepsilon$, and was chosen as $\alpha=2$. Such parametrization of the description of shear deformation gives the advantage of simple building of shear curves based only on the initial fabric parameters: its lineal density and looseness, and the pretension. The performed parametric study revealed the increase of the shear resistance with the decrease of $s$ and the increase of $t$ and $\varepsilon$, being especially sensitive to the fabrics pretension $\varepsilon$ in picture frame shear. Compared to the experimental, the constructed curves showed good agreement up to the locking shear, and less steep form after locking, explained, firstly, by the influence of the approximate description of lateral compression in the model in [39], and thus in Eqs. (11); and, secondly, by the possible increase of tension on the frame during the test, not accounted for. 


\section{FEA continuous}

The continuum finite element models represent a large class of models that describe the shear deformation of fiber materials. The majority of models were developed for woven fabrics. A part of them can be related to the mesoscopic scale [45-50]. In the continuous formulation, the woven fabric is seen as an anisotropic planar continuum with two principal material directions (Fig. 15a). Depending on the formulation of the model within the FEA framework and the underlying mesoscopic phenomena taken into account, the model is able or not to address large shear deformations. Besides, different FEA continuous models may require different additional tests to be performed for the inverse identification of input parameters for the model.

The developments of the continuum non-orthogonal model by Xue et al. [28], mentioned in Section "Macroscopic (whole component) scale ", were extended to address the mesoscopic scale as well [45]. The micromechanical model describing large shear deformation of the woven cell, and accounting for the yarn compaction during shear, was integrated into the continuum FE shell elements. The yarn compaction was introduced in the calculation of the stiffness matrix of the unit cell via the relation for the yarn width $w$ : $w=w_{0}(\sin \vartheta)^{\alpha g_{0} / w_{0}}$, where $w_{0}$ is the initial yarn width, $g_{0}$ is the inter-yarn gap, $\vartheta$ is the angle between warp and weft yarns, and $\alpha$ is the empirical coefficient. The uncoupled shear and tensile properties in the global non-orthogonal Eq. (3) were defined this time from the micromechanical model, with input structural and material properties of yarns, not by experimental matching as in [28]. The comparison of the total force-displacement dependence of the picture frame shear deformation with the experimental one showed good agreement. It was also found that at later stages of deformation the shear stiffness of the material increases with the increase of the Young's modulus of the fibers, as well as with the increase of the fiber packing fraction, and with the decrease of the inter-yarn gap.

King et al. [46] performed extensive continuous FE analysis to model macroscopic in-plane deformations of plain woven fabrics with the incorporated mesostructural state of yarns deformation. The woven RUCs configuration was defined on the basis of Kawabata's et al. model [42] (Fig. 15b) with the addition of truss elements, which remain normal to the yarns and allow to account for their locking in shear.

With the assumptions of constant fabric thickness and non-sliding of yarns at the cross-overs, King et al. addressed eight meso-scale modes: four of them describing linear elastic resistance to extension and to bending of the two (warp and weft) yarn families; cross-sectional compression at cross-overs defined by the non-linear interference spring (Fig. 15b); locking at cross-overs; linear elastic relative yarn rotation; and dissipative yarn rotation defined as a rate-dependent power law. The fabric configuration was then identified from the macroscopic deformation gradient by minimizing a conditional elastic energy function, and the RUC internal forces were calculated. The latter were divided by the projected areas to obtain continuum macrostresses. The needed input yarn geometrical and material properties were measured in separate tests. The proposed approach gave good predictions of the shear angle distributions and load-displacement relations of the bias extension deformation.

The continuum FE formulation was also used by Lin et al. [51] to describe large deformations of woven fabrics subjected to the combined shear and compression loading, which is poorly covered in the literature. At the same time, namely a combination of loads, composed of fabric shear for conforming to the doubly-curved shapes, and compression due to the tool closure before the injection of matrix, is encountered during forming of the material to obtain composite parts. To address the non-linear material behavior in large deformations, the non-linear transverse mechanical law: $E_{33}\left(\varepsilon_{33}\right)=\sigma_{33} / \varepsilon_{33}=\frac{-a\left(V_{f 0} / e^{\varepsilon_{33}}\right)^{b}+a V_{f 0}^{b}}{\varepsilon_{33}}, G_{23}=$ $\frac{E_{33}}{2\left(1+v_{23}\right)}$ was incorporated in the stiffness matrix with nine independent constants. $E_{33}$ is the transverse Young's modulus; $G_{23}$ is the transverse-longitudinal shear modulus; $V_{f 0}$ is the initial fiber volume fraction; $a$ and $b$ are fitting the yarn compression test data parameters. It was found that different $V_{f 0}$ affected both compressive and pure shear behavior of fabrics (the analogous to [45] conclusion), making them dependent on the transverse Young's modulus. Coupling of loads showed the need to apply higher shear forces in order to deform the compacted material to the same degree as non-compacted.

Some investigations concerning the adequacy of the mesoscopic continuous FE approaches in modeling yarns deformation within the material were made by Durville [52]. With the assumption of the continuity of yarns, the author calculated Green-Lagrange strain-tensors by the 3D finite elements with the mesh based on the nodes defined on fibers. The strong inhomogeneities in strains were observed in simulations of the equibiaxial tension, for example: the obtained shear strains meant a rearrangement of fibers in the center of the yarn; the axial strain was found to be twice higher on the sides of the yarn than in the center.

Therefore, in order to use the advantages of the continuous FE approach and to overcome its drawback in the prediction of large non-linear shear deformations of fibrous materials, Boisse et al. in [47], Badel et al. in [48-50] employ the hypoelastic model with the specific objective derivative, used to update the orientation of the constitutive tensor, as long as it changes owing to the fiber rotations within the yarn. In [50] it was shown that the models based on objective derivatives of Green-Naghdi or Jaumann 

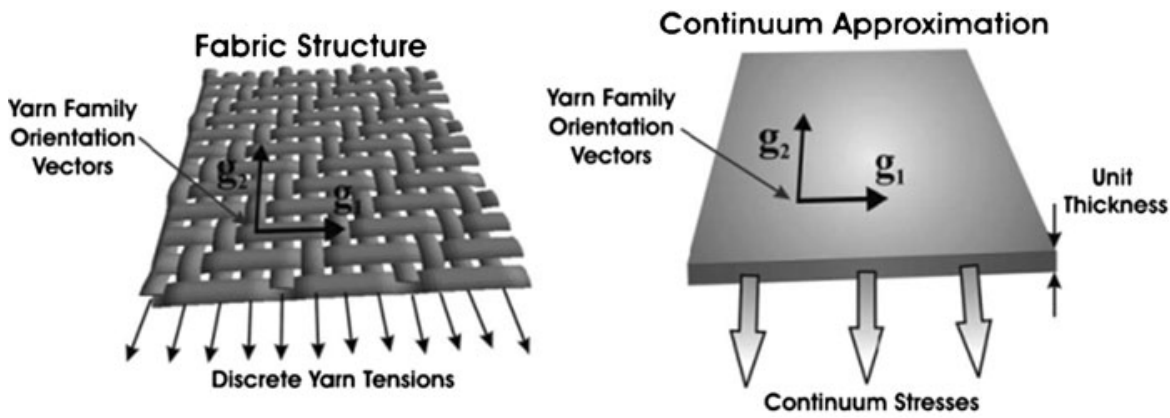

a)

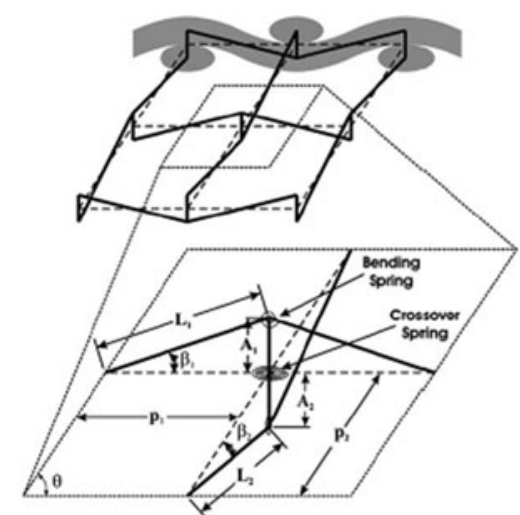

b)

Fig. 15 a Approximation of a fabric as an anisotropic continuum; b geometry model of the woven RUC [46]

describing the average rotation of the continuum do not give adequate response. The proposed constitutive relation $\underline{\underline{\sigma}}^{\nabla}=\underline{\underline{\underline{C}}}: \underline{\underline{D}}$ of the stress rate $\underline{\underline{\sigma}}^{\nabla}$ to the strain rate $\underline{\underline{D}}$ by the elastic tensor $\underline{\underline{\equiv}}$ is based on the rotation $\triangleq$ of the fiber. Through cumulating in the rotated frame, this results in the following stress tensor:

$\underline{\underline{\sigma}}=\underline{\underline{\Delta}} \cdot\left(\int_{0}^{t} \underline{\underline{\Delta^{T}}} \cdot(\underline{\underline{\underline{C}}}: \underline{\underline{D}}) \cdot \underline{\underline{\Delta}} d t\right) \cdot \underline{\underline{\Delta^{T}}}$

The initial orthotropic axes $\left\{k^{0}\right\}$ along the elements are redefined to the current physical constitutive axes $\left\{k^{t}\right\}$ as follows:

$\underline{k}_{i}^{t}=\underline{\underline{\Delta}} \cdot \underline{k}_{i}^{0} ; \quad \underline{k}_{1}^{t}=\frac{\underline{F} \cdot \underline{k}_{1}^{0}}{\left\|\underline{\underline{F}} \cdot \underline{k}_{1}^{0}\right\|}, \quad \underline{k}_{2}^{t}=\underline{k}_{2}^{0}-\frac{b_{2}}{1+b_{1}}\left(\underline{k}_{1}^{0}+\underline{k}_{1}^{t}\right)$,

$\underline{k}_{3}^{t}=\underline{k}_{3}^{0}-\frac{b_{3}}{1+b_{1}}\left(\underline{k}_{1}^{0}+\underline{k}_{1}^{t}\right)$,

where $b_{k}=\underline{k}_{1}^{t} \cdot \underline{k}_{k}^{0}$ and $b_{k} \neq 1, \mathbf{F}$ is a deformation gradient. The contact between yarns was also included in the analysis by the master/slave technique with Coulomb friction. The adequacy of the model was demonstrated by the good correlation of the shear load-displacement curves for the woven unit cell to the experimental ones [47, 48, 50]. In [47] an implicit scheme gave good values until the locking angle. The appeared at this stage convergence problems were suggested to be solved by using an explicit scheme, or by the local mesh refinement. In [48], in addition, the comparison of predictions for two RUC forms was made in order to correctly address the periodicity and boundary conditions. In [49] the model was improved by uncoupling the "spherical" (though in 2D meaning) and the deviatoric phenomena in the transversely isotropic behavior of yarns, the first describing the fiber density changes within the yarn, the second accounting for cross-section shape changes. This allowed to obtain the laterally compacted (as a result of pure shear) yarn cross-sections well-agreed with X-ray tomography cross-sections shapes [49].

In [50] and later, in [53], the hypoelastic model was extended to calculate the macroscopic shear deformations of the fabric in hemispherical forming. Since this macroscopic approach chronologically originates from the mesoscopic hypoelastic approach, for the sake of clarity its essence and predictions, made by it, will be described here, in the section devoted to the mesoscopic scale. For the situation of this method in the classification, the reader is referred to Fig. 2.

The principle of the macro-approach is the superposition within the elementary material volume of two stress states (sum of tension and shear) for two yarn directions of woven fabrics, each of which is obtained similarly as in the meso-approach [50]. This superposition represents the sum of the transformed stresses calculated in the two orthogonal rotated frames containing fiber directions, and expressed then in the Green-Naghdi's frame [53]. The check of validity of the approach on the elementary tests of single element analyses (simple shear test, tension followed by the simple shear, and by the rotation) has shown that it is capable of predicting correctly large strains. In order to perform macroscopic forming simulations for woven fabrics, the approach required as the parameters the elastic tensile moduli for two yarns directions, the in-plane shear rigidity (obtained by the inverse method), the friction coefficient, and the blank holder force. A good correlation with experimental data was observed for the shear angle distributions and deformed fabric shape for $0^{\circ} / 90^{\circ}$ and $\pm 45^{\circ}$ yarn orientations in the hemispherical forming [50], and in the double dome benchmark forming [53]. Besides, multilayer fabrics forming simulations were performed with the satisfactory convergence [53]. 


\section{FEA semi-discrete}

Although a number of continuous FE models, with different level of complexity and accounting for different factors of the shear behavior of fibrous reinforcements, were developed, the alternative to these approaches was suggested recently in order to avoid the difficulties of continuous modeling of large deformations of highly anisotropic materials. The suggested semi-discrete FE approach [5, 6, 54, 55] is close to the discrete approach in the sense that finite elements are composed of discrete number of constituents yarns, unit cells (RUCs) etc., however, it prevails over the discrete approach thanks to the avoidance of large number of unknowns. Boisse et al. [5], Zouari et al. [54], Hamila and Boisse [6, 55], within the framework of semi-discrete method, demonstrated the importance of addressing the shear rigidity in the analysis of large deformations of woven fabrics by expressing its interior virtual work by three terms in a dynamic equation as follows:

$$
\begin{array}{r}
\sum_{p=1}^{n c e l l} \int_{p_{l}}{ }^{p} T^{11 p} \varepsilon_{11}(\underline{\eta}) \mathrm{d} l+\sum_{p=1}^{n c e l l} \int_{p_{l}}{ }^{p} T^{22 p} \varepsilon_{22}(\underline{\eta}) \mathrm{d} l \\
\quad+\sum_{p=1}^{n c e l l}{ }^{p} C_{s}{ }^{p} \gamma(\underline{\eta})-W_{\text {ext }}(\underline{\eta})=\int_{\Omega} \rho \underline{\ddot{u}} \cdot \underline{\eta} \mathrm{d} V
\end{array}
$$

$\forall \underline{\eta} / \underline{\eta}=0$ on $\Gamma_{u}$, where ncell is the number of the unit woven cells in the domain under consideration $\Omega ; \varepsilon_{11}(\eta)$ and $\varepsilon_{22}(\eta)$ are the virtual axial strains in the warp and weft directions; $\gamma(\eta)$ is the virtual angle between warp and weft directions; $T^{\overline{11}}$ and $T^{22}$ are the axial tensions on the unit woven cell; $C_{S}$ is the shear torque; $\underline{\ddot{u}}$ is the acceleration of the point $P$; $\rho$ is the mass per volume; $\Gamma_{u}$ is the part of the boundary with the prescribed virtual displacement field $\eta ;{ }^{p} A$ means that the quantity $A$ is considered for the cell number $p$. The mesoscopic material properties $T^{11}, T^{22}, C_{S}$ were input in the model either from the elementary tests $[5,54,55]$, or from the $3 \mathrm{D}$ computations of the unit woven cell $[5,6]$. Thus the friction between yarns (master/slave technique in case of $3 \mathrm{D}$ cell) as well as transverse yarn compaction during shear were anyhow accounted for in the analysis. However, the drawback of inputs from the tests is that the change in yarns undulations during deformation is not taken into account in this case [55]. It should be noted that the semi-discrete approach occupies an intermediate place between the macro- and meso-scale approaches. Since in $[5,6,54,55]$ meso-scale properties were incorporated in the semi-discrete elements and influenced the simulated results, these works are related here to the predictions made at the mesoscopic scale.

The peculiarity of the semi-discrete approach is defining tensile and shear strains as functions of nodal displacements of each element, accounting for the interpolation of the geometric and kinematic conditions within the element (4-node [5, 54] or 3-node [6, 55] - see Fig. 16a). Thus the condition of continuity, i.e. that the yarns at cross-overs do not slide, is imposed in the approach. The formulation of 3-node FE, as opposed to 4-node, does not restrict the directions of components to be aligned with the sides of the element providing the advantage for the mesh refinement and remeshing.

The performed analysis of the forming of one ply and five plies of fabric over a hemisphere showed good agreement with experiments. Besides, large deformations in forming over square box, cubical and circular table showed wrinkles apparition as in reality only with the shear stiffness included (Fig. 16c), unlike the zero-shear cases (Fig. 16b).

It should be noted that in continuous and semi-discrete FEM simulations of fibrous materials the numerical problem termed intra-ply shear locking (not to be confused with the physical phenomena of material shearing) may arise. It was initially identified by $\mathrm{Yu} \mathrm{X}$. et al. in 2005 (extended in [56]). The incorrect predicted deformation profiles as well as the so-called spurious wrinkles can be observed during woven fabric bias extension simulations and forming simulations by the shell finite elements $[56,57]$. They represent the consequence of the incorrect element displacement field and overestimated stiffness, when the intra-ply shear modes of 4-node or 3-node elements, depending on mesh orientation, are not fully addressed. On the way to overcome this problem the effectiveness of aligning element boundaries with fiber/yarn directions at the beginning of simulation was shown in [56] while simulating 2D bias extension and 3D diaphragm forming. On the other hand, aligning the mesh restricts the use of automatic mesh generation, and does not allow to use multi-layer elements [57]. Therefore, in the case of unaligned meshes [57] proposed a way out as the employment of the triangular multi-field membrane element, because it owns a semi-quadratic in-plane and a linear out-of-plane displacement field. The use of selective reduced integration, implying that only the fiber components of the material model are under-integrated, was proposed to help to improve the accuracy of simulations in case of arbitrary meshes as well.

Non-crimp/stitched materials

\section{Continuous energy based approach}

In comparison to the wovens, at mesoscopic scale the shear behavior of NCF additionally includes intricate stitch-toyarn interactions, stitch tension and inter-stitch friction at loop points [58], which cannot be modeled very accurately by macroscopic models. 


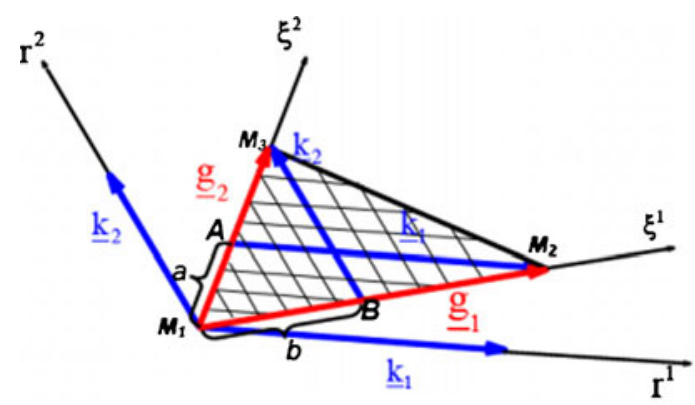

a)

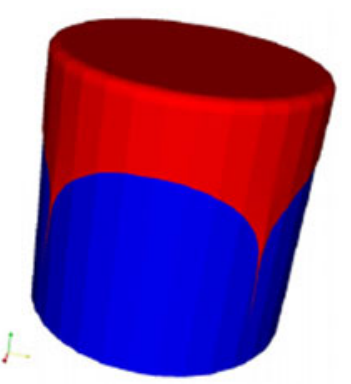

b)

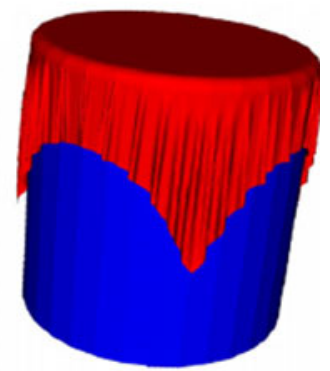

c)

Fig. 16 a 3-node finite element made of fibrous reinforcement components; b woven fabric draped over a circular table without shear stiffness and $\mathbf{c}$ with shear stiffness [55]

Therefore, Wiggers et al. [58] addressed the stitch effects, as well as the yarns compaction in their mesoscopic energybased model for the tricot-stitch warp-knitted $\pm 45^{\circ} \mathrm{NCF}$, however, without accounting for the inter-yarn friction, and neglecting the interaction between the stitch tension and the yarn compaction. Thus the application of the model is limited to low shear angles, i.e. without the variation of fabric thickness $t$. Stitch tensile deformations were determined out of the modified length of the stitch segments $c, d$ and $t$ (Fig. 17a) expressed by the stitch length $c_{0}$, stitch spacing $h_{0}$ and a shear angle basing on the geometrical reasoning (Fig. 17b). Other required input parameters were the stitch modulus, the stitch effective cross-sectional area, and the fabric size.

The inter-stitch friction was defined by considering the lateral force at the point of contact as being equal to the stitch tension. The tow compaction description was adopted from the one by Cai and Gutowski [59]. Hence the predicted shear force-angle curves successfully showed the asymmetric shear behavior of NCF, depending on the load direction, and also on the stitch length. However, the curves go to infinity in a steeper way and at a smaller angle than the ones from the picture frame test, that was attributed to the $t=$ const assumption (Fig. 17a).

\section{FEA discrete}

Later, the employment of the discrete finite elements approach allowed Creech and Pickett [18] to address the shear deformation in draping of the macroscopic NCF parts (analogically, tricot-stitch warp-knitted $\pm 45^{\circ} \mathrm{NCF}$ ), and accounting for the mesoscopic effects mentioned earlier (Fig. 18).

A mesoscopic representative cell of the model (Fig. 19) consisted of two layers, constructed of solid elements to represent the yarns (tows), and of bar elements to represent the tricot stitch. Unlike [58], Creech and Pickett included the stitch-to-yarn interactions (Fig. 18g) in their analysis by defining the friction forces from the tow pullout test. Besides, the inter-tow friction, previously ignored, was modeled by Coulomb friction coefficients, measured after being separated into three cases: sliding between tows of different plies, sliding between tows of the same ply, and cross-over point sliding (Fig. 18b, c and d respectively). An assumption was made that the shear deformation until $\approx 30^{\circ}$ was dominated by stitch effects (Fig. 18e, f), and after - by the tow compaction (Fig. 18a). The stitch stiffness was identified by the analytical formula from [58], while the tow lateral compaction - by the inverse identification from the picture frame test. Consequently, the simulations of the

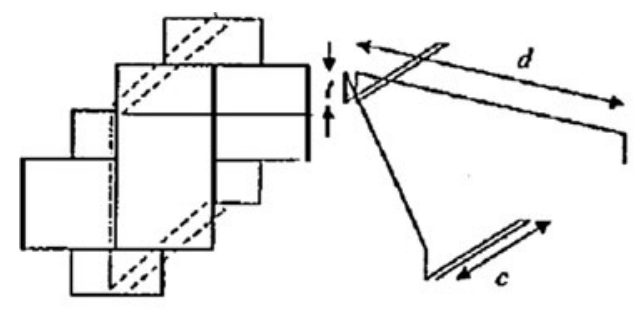

a)

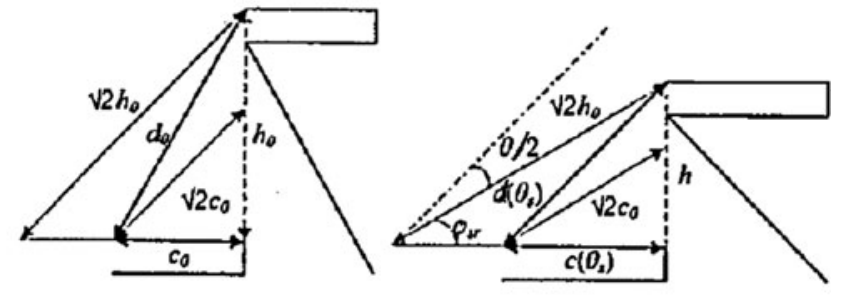

b)

Fig. 17 a Tricot-stitch warp-knitted $\pm 45^{\circ}$ NCF unit cell (left) and its stitch (right); b scheme of the unit stitch before and after shear [58] 


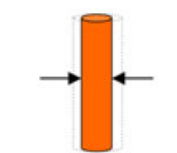

Tow compaction

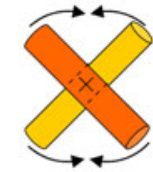

Inter-tow shear

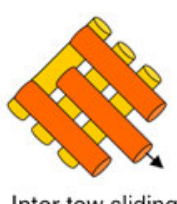

Inter-tow sliding

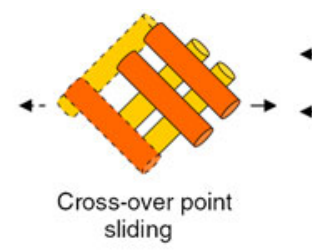

d)

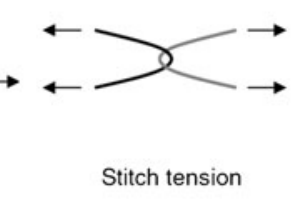

e)

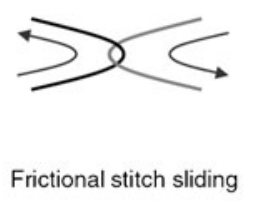

f)

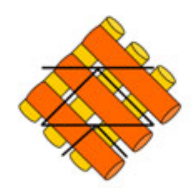

Interaction between stitching and fibre tows

a)

b)

c)

Fig. 18 Mesoscopic deformation mechanisms constituting shear deformation of non-crimp fabric [18]

bias extension gave good agreement between experimental and predicted force-strain curves and angle distributions, as well as in yarn slippage zones. An important achievement was the capability of the model to reproduce the asymmetrical deformation of the NCF during hemispherical forming, moreover, with high accuracy.

\section{FEA semi-discrete}

The developed initially for woven fabrics semi-discrete approach has been recently employed by Bel et al. [60] for the prediction of deformations during forming of $+90^{\circ} / 0^{\circ}$ biaxial warp-knitted NCF. Each of UD fiber layers was modeled by triangular shell elements whose internal forces contain the tensile, bending moment and shear moment (perpendicular to the shell) components. The corresponding constitutive equations of fiber layers were assumed to be linear elastic because all non-linearities were supposed to originate from sliding between stitches and fibers. The input values of tensile, bending, and shear stiffness were obtained by the inverse identification. One of the main objectives of the approach was addressing the inter-ply sliding observed during non-crimp fabrics forming operations in [60, 61]. The inter-ply sliding implied the distance between the projection on the mid-surface of the NCF of two points initially opposed and belonging to different plies. Large sliding was modeled with the help of bar elements (simply meshed) connecting fiber layer shell elements. The anisotropic Coulomb friction was considered between the bar and the shell elements, allowing sliding between layers and between layers and stitches, but only in the fiber direction for the latter. Sliding in other directions was neglected because the tows of NCF are supposed to be tightly hold by interstitching. Hence the approach accounts for all listed above

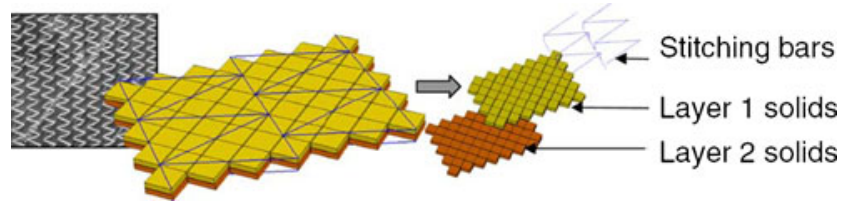

Fig. 19 A representative unit cell of the meso-mechanical model for the non-crimp fabric [18] mesoscopic mechanisms contained in the shear deformation of non-crimp fabrics, except for the frictional inter-stitch sliding (Fig. 18f). It additionally includes the out-of-plane bending of fiber layers inherent in preforming.

As a result, the prediction of hemispherical forming showed close to the experimental observations inter-ply sliding, almost negligible at the top of the hemisphere, and reaching the maximum of approximately $14 \mathrm{~mm}$ far from the top (Fig. 20). Observed in both (perpendicular) directions, sliding is found to be symmetric for each of them with respect to the top, and different for two directions, which is attributed to the difference in bending stiffness in these directions. Therefore, the advantage of the developed approach is its capacity to adequately predict the deformations of large parts made out of NCF with reasonable computational time. Another advantage is that the employment of bar and shell elements does not restrict varying the mesh size, whatever the fabric unit cell size is. The inconvenience of the approach is the necessity to perform additional tests for the definition of local rigidities.

\section{Microscopic (fiber) scale}

The consideration of shear deformations of fibrous materials at microscopic scale is known in the literature particularly for woven fabrics. The changes of the yarn crosssection shape associated with shear are caused by microscopic rearrangements of fibers inside the yarns. Besides, the global shear response of the fabric is influenced by presence of contacts and friction between fibers at microscale. So modeling at this scale should take into account the aforementioned phenomena. The predictions, however, are complexified by the heterogeneous structure of the material at micro-scale where the repeated structural cells can hardly be defined, as opposed to meso-scale.

Extended geometrical approach

Hofstee et al. [62] proposed to simulate the shear deformation of woven fabrics by the help of fishnet approach, described earlier, but replacing the fishnet segments (assumed to be inextensible sections, representing yarns, 


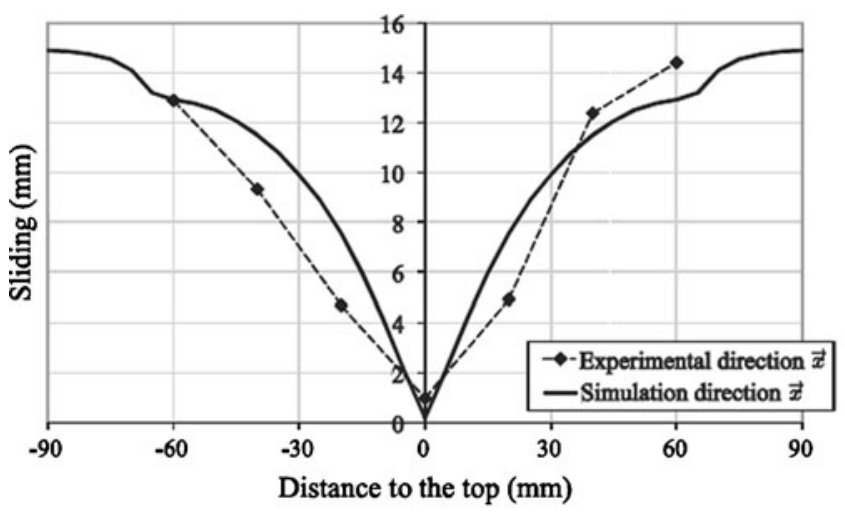

a)

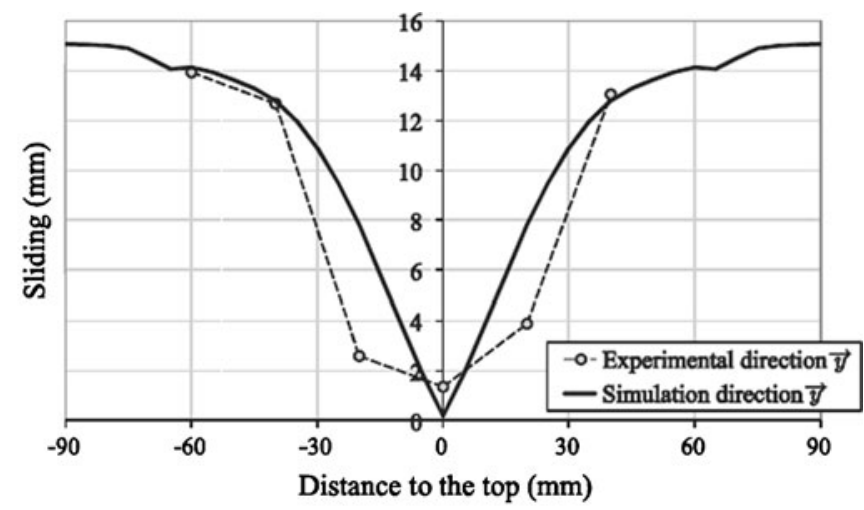

b)

Fig. 20 Sliding in the two main directions of the square sample for the experiment and the simulation according to the direction $\mathbf{a} \vec{x}$; $\mathbf{b} \vec{y}$ [60]

connected by pivots) with the 3D RUC of typical crimped pattern (Fig. 21). The yarns, or fiber bundles, in the RUC are described by the in-plane centerline path, curved horizontal midplane, and variation of cross-sectional thickness, with initial values prescribed from the experimental observations and cross-section photomicrographs. Constant packing and constant position with respect to each other were assumed for fibers inside a bundle. Besides, the moderate deflection angles were assumed between the local and mean fiber bundle orientation. The RUC shear deformation, which was considered as a combination of 1) yarns out-of-plane undulation, 2) midplane curvature and 3) midplane twist, was modeled as a loading imposed by the hinged frame.

The predicted deformed geometries of the plain-weave balanced and unbalanced fabrics showed the increased yarn thickness, as similarly observed on the laminate crosssection photomicrographs. This is a result of the decreased distance between yarns, the reduction of yarns width, and consequence of constant volume condition. Good agreement was observed between the photomicrographs and simulated sheared to $54^{\circ}$ configurations of balanced fabric, as well as between the sheared to $61^{\circ}$ unbalanced fabric and corresponding photomicrographs. The simulation also showed good agreement between cross-sections normal to the mean yarn direction, where twisting was successfully captured by the model. The shear prediction of other types of wovens (twill, satin) is possible with the proposed approach too.

\section{FEA discrete}

The discrete finite elements approach developed for the simulation of different fibrous materials (Fig. 1) deformation at microscopic scale by Durville in [63] was also applied to model shear deformation of woven fabrics [64-66]. The approach is based on the 3D-beams theory where the crosssections of beams are described by three vector fields: one for the translation of the beam centroid, two others for the orientation and deformation of the cross-section. This represents an improvement with respect to the classical beam models with rigid cross-sections, because the deformed beams' trajectories can be simulated without complex formulations for large rotations, typical for the beam models described by two vector fields. The problem is set as a

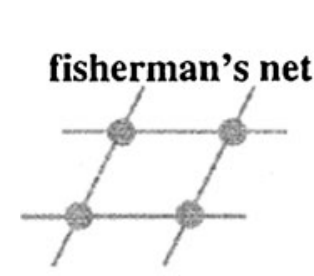

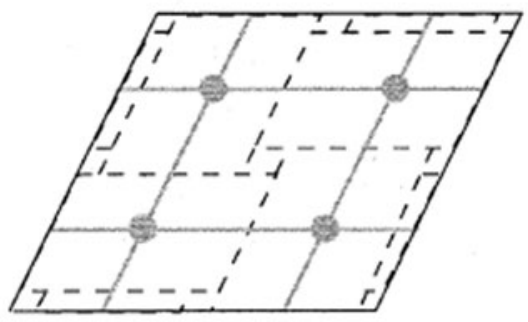

a)

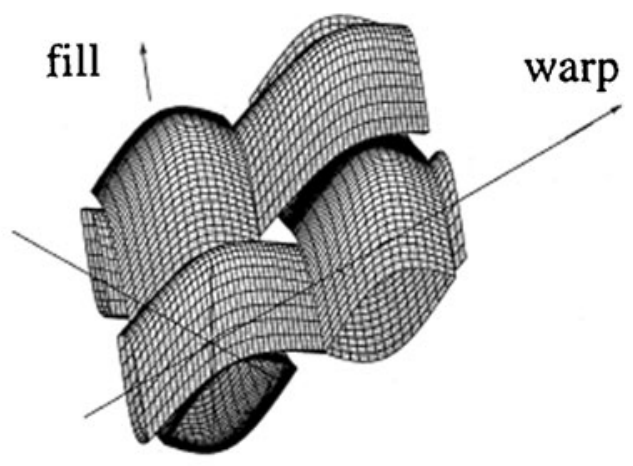

b)

Fig. 21 a Extended fishnet with b the 3-D geometry model of its unit cell - sheared configuration [62] 
principle of virtual work, which includes the virtual works of all beams, and the virtual work of contact-friction interactions between fibers. The virtual work of internal forces for each fiber is expressed using a total Lagrangian formulation:

$W_{i n t}^{(i)}(U, V)=\int_{\Omega(i)_{0}} \operatorname{Tr}\left(s(U) \frac{D E}{D U} \cdot V\right) d \omega$

where $U$ is the generalized displacement field, $V$, the corresponding virtual field, $E$ is the Green-Lagrange strain tensor, and $s$, the second Piola-Kirchhoff stress tensor.

At first, the initial crimped fabric configuration was defined by simulating the weaving process basing only on the description of weaving pattern. An important issue here is the provision of "flexible" boundary conditions, which while applying global loadings to the end of each component (edge of the sample or yarn) could allow the subcomponents (yarns for the edges or fibers for the yarns) to move and rearrange. It was realized by attaching rigid bodies to the ends of fibers and yarns and usage of average binding conditions (on average displacements and average rotations of subcomponents). The condition on the displacement is expressed so that the displacement of the barycenter of nodes of subcomponents is the same as the displacement of the rigid body, with respect to the moving frame attached to the rigid body. The condition on the average rotation prevents the rotation of the set of subcomponents around three directions of the rigid body. Consequently, each of these rigid bodies can be driven either by displacement/rotation, or by force/moment.

After obtaining the initial configuration of the plainweave fabric, two opposite edges of the latter were subjected to opposite horizontal displacements, while displacements in orthogonal directions were left free, as well as two remaining edges of the fabric. Hereby the

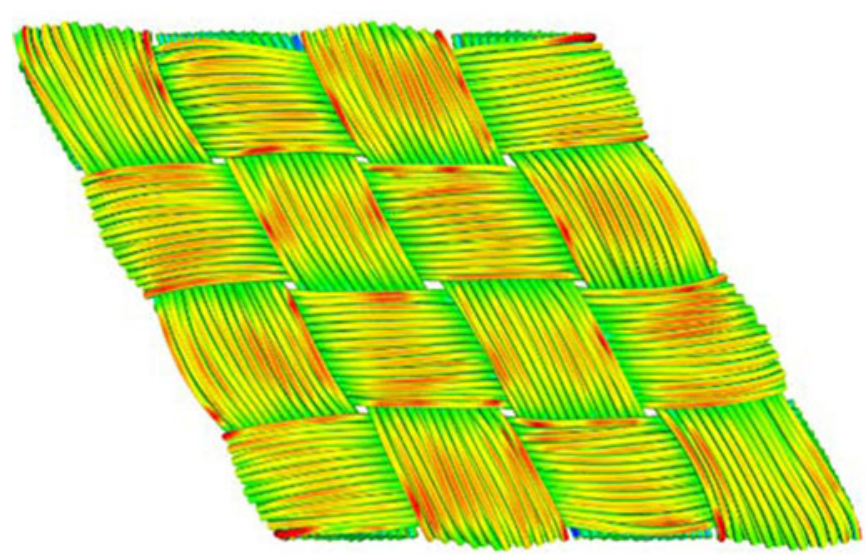

a) loading/unloading/re-loading cycle, up to the shear angle of nearly $25^{\circ}$, was simulated (Fig. 22).

The shape of the loading curve agrees well with the one obtained in the known benchmark results [11]. The same non-linearity due to the locking between yarns, and hysteresis associated with the friction between fibers (friction coefficient 0.2) are observed. However, the convergence problems of the model restrict the maximum attainable shear angle. Although the "flexible" boundary conditions bring the model nearer to the reality, they probably need to be improved, especially at the corners of the sample at the level of fibers, to reduce convergence issues.

\section{Conclusions}

A summary of the existing methodologies for the prediction of fibrous materials response to the shear loading, inherent in forming procedures, has been presented. The approaches have been classified primarily by the scale, at which they address the shear properties whether of the whole material part, or taking into account the constituents - yarns and/or fibers. The classifications according to the fibrous material architecture being modeled, and according to the theoretical framework employed, have been proposed as well. The variety of approaches in this domain, and the absence of the generally accepted one, is attributed to the intricate structure of fibrous materials causing their strongly anisotropic behavior.

Forming of particularly two classes of fibrous materials is realized primarily by the shear strains: woven and noncrimp fabrics. Hence the models addressing the specific shear behavior of each of these classes have been developed. The shear deformation can be investigated with the help of

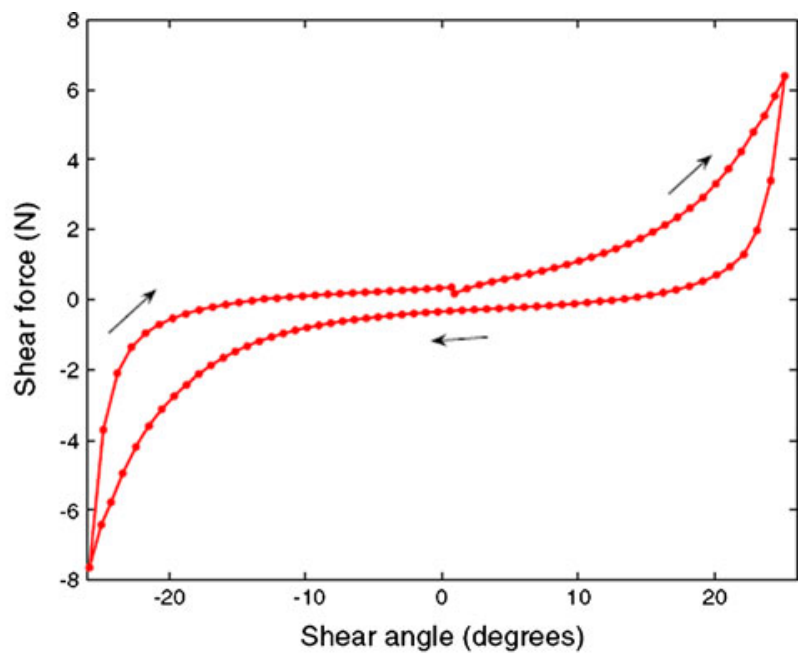

b)

Fig. 22 Configuration at the end of the simulation of the shear test (left) and loading/unloading curves (right) for the plain woven fabrics [66] 
the three characterization tests: picture frame, bias extension, and simple shear. In order to validate the predicted shear deformation results, the practical realization and simulation of forming to the hemisphere, as the most widely used shape, can be performed. Modeling of the simple shear mode is of particular interest because it is difficult to realize it experimentally maintaining the necessary boundary conditions. While comparing the predicted picture frame shear and the one obtained in the test, the pretension of fabrics, present in the test, should be taken into account. Theoretical prediction of bias extension of woven fabrics should give as a result typical for wovens distinct zones with the corresponding strain magnitudes.

For the non-crimp fabrics the approach should be able to predict its assymetric (as opposed to the majority of woven fabrics) shear behavior in forming dependent on the direction of load application. And here the macroscopic geometrical, including geometrical in combination with the energy principle, as well as FEA continuous models gave satisfactory qualitative results. It is found that the assymetry in the NCF deformation can be diminished by increasing the blank-holder force, as well as by increasing the friction effect. The most accurate results are obtained by the approaches taking into account all the characteristic for the NCF deformation mechanisms: stitch-to-yarn interactions, stitch tension, and inter-stitch friction at loop points. Therefore, the models particularly at meso-scale are capable of doing that: the energy based one, the FEA discrete and FEA semi-discrete. The first two deformation mechanisms can be omitted, still giving realistic predictions by the energy based approach, if the initial stages of shear deformation only are under consideration. FEA discrete and semi-discrete approaches have been shown to be the most precise in addressing the shear behavior of NCF up to large deformations.

There is even larger variety of approaches developed to predict shear properties of woven fabrics: geometrical, energy based, force based, empirical, FEA continuous, FEA discrete, and FEA semi-discrete. Nowadays geometrical methods are still widely used as a quick design tool in industry due to its minimal requirements of data input and specification of the shape. A number of commercial codes, such as CATIA CPD, PAM-QUIKFORM, FiberSIM, MSC Laminate Modeller, use them. On the one hand, the advantage of these methods is their simplicity in realization, and they can give quite adequate results (once the net size is refined enough) for the initial fabric deformation stages, as was proved by experiments and sensitivity analysis of the node location versus the net size. On the other hand, in order to describe the later stages of deformation, which lead to the onset of wrinkles (buckling) after a certain shear angle value is reached, the models require additional information from experiments, at least the locking angle value as an input. However, including this parameter in the macroscopic geometrical or continuous FE model still does not give a sufficient accuracy in the prediction of the later stages of shear deformation. It is attributed to the fact that at these stages the material behavior is greatly determined by its architecture, i.e. yarn sizes, yarn path, yarn spacing, as well as by the mechanistic effects, e.g. inter-yarn friction, buckling resistance. These factors prompt to model the material at mesoscopic scale.

Mesoscopic continuous FE models with the measured aforementioned mesoscopic parameters, input in the analysis, give reasonable predictions of the shear behavior of woven fabrics. The parametric studies have shown that the shear stiffness is higher if the Young modulus of yarns is higher, and if the yarn spacing is decreasing. The continuous FE model has also handled the analysis of the combined shear and compression loads, typical for the shaping procedure, and has shown that preliminarily compacted material is more difficult to shear than the uncompacted one. The limitations of the continuous FEA show up in the identification of homogenized material parameters, because these parameters alter when the directions of the yarns change and lateral contact between the yarns occurs due to large strains. Employment of the objective derivative following the fibers in their rotation within the yarns in the hypoelastic continuum FE model has allowed to get rid of these limitations, and to give good results.

Another efficient alternative to avoid the continuity limitations imposed in the continuous FE models is to employ the recent semi-discrete FE approach, whose finite elements are composed of mesoscopic constituents - yarns. In order to omit computational difficulties of numerical approaches, the force based or empirical approach, accounting for the happening at meso-scale phenomena such as yarns lateral compression, inter-yarn friction, can be used for fast calculations of the shear deformation. The energy-based approach allows to define the most important mesoscopic mechanisms that contribute the most to the total energy at each of the shear deformation stages.

At micro-scale FEA discrete approach successfully models fabrics and its shear deformation starting from its constituents that are continuous indeed - fibers, their contact/friction interaction, up to the whole component structure. The inconvenience of the approach is that for the realistic results it demands significant computational costs.

\section{References}

1. Peirce F (1930) J Text Inst 21:377

2. van Wyk C (1946) J Text Inst 37:285

3. Abbott N (1951) Text Res J 21(6):435 
4. Vidal-Sallé E, Boisse P (2009) Modelling and predicting textile behaviour (Taylor and Francis), chap. Modelling the structures and properties of woven fabrics, pp 144-179

5. Boisse P, Zouari B, Daniel J (2006) Compos A Appl Sci Manuf 37:2201

6. Hamila N, Boisse P (2007) Appl Compos Mater 14(4):235

7. Dumont F, Hivet G, Rotinat R, Launay J, Boisse P, Vacher P (2003) Mèch Ind 627-635

8. Lomov S, Huysmans G, Luo Y, Parnas R, Prodromou A, Verpoest I, Phelan F (2001) Compos A Appl Sci Manuf 32(10):1379

9. Dumont F (2003) Contribution à l'expérimentaion et à la modélisation du comportement mécanique de renforts de composites tissés. Ph.D. thesis, Université d'Orléans, Université de Paris 6, France

10. Lomov S, Barburski M, Stoilova T, Verpoest I, Akkerman R, Loendersloot R, ten Thije R (2005) Compos A Appl Sci Manuf 36(9): 1188

11. Cao J, Akkerman R, Boisse P, Chen J, Cheng H, de Graaf E, Gorczyca J, Harrison P, Hivet G, Launay J, Lee W, Liu L, Lomov S, Long A, de Luycker E, Morestin F, Padvoiskis J, Peng X, Sherwood J, Stoilova T, Tao X, Verpoest I, Willems A, Wiggers J, Yu T, Zhu B (2008) Compos A Appl Sci Manuf 39(6):1037

12. Hu JL, Teng JG (1996) Finite Elem Anal Des 21:225

13. Ng HN, Grimsdale RL (1996) IEEE Comput Graph Text Appar 16:28

14. Pickett A, Creech G, de Luca P (2005) Eur J Comput Mech 14:677

15. Boisse $P$, Hamila N, Helenon F, Hagège B, Cao J (2008) Int J Mater Form 1:21

16. Boisse $\mathrm{P}$, Aimène $\mathrm{Y}$, Dogui A, Dridi S, Gatouillat S, Hamila N, Aurangzeb Khan M, Mabrouki T, Morestin F, Vidal-Sallé E (2010) Int J Mater Form 3:1229

17. Syerko E, Comas-Cardona S, Binetruy C (2012) Compos A Appl Sci Manuf 43(8):1365

18. Creech G, Pickett A (2006) J Mater Sci 41(20):6725

19. Peng X, Cao J (2005) Compos A Appl Sci Manuf 36(6):859

20. Aimène $Y$, Vidal-Sallé E, Hagège B, Sidoroff F, Boisse $P(2010)$ J Compos Mater 44(1):5

21. Yu W, Harrison P, Long A (2005) Compos A Appl Sci Manuf 36(8): 1079

22. Potter K (1980) Controller. HMSO, London, pp 1564-1579

23. Robertson R, Hsiue E, Sickafus E, Yeh G (1981) Polym Compos 2:126

24. Bergsma O, Huisman J (1988) In: Brebbia C, de Wilde W, Blain $\mathrm{W}$ (eds) 2nd international conference on computer aided design in composite material technology. Springer Verlag, Southampton, pp 323-333

25. Prodromou AG, Chen J (1997) Compos A Appl Sci Manuf 28(5):491

26. Long A (2001) 4th international ESAFORM conference on material forming, pp 99-102

27. Sickafus EN, Mackie NA (1974) Acta Crystallogr A 30(6):850

28. Xue P, Peng X, Cao J (2003) Compos A Appl Sci Manuf 34(2): 183

29. Yu W, Pourboghrat F, Chung K, Zampaloni M, Kang T (2002) Compos A Appl Sci Manuf 33(8):1095

30. Aimène $\mathrm{Y}$, Hagège $\mathrm{B}$, Sidoroff $\mathrm{F}$, Vidal-Sallé $\mathrm{E}$, Boisse $\mathrm{P}$, Dridi $\mathrm{S}$ (2008) Int J Mater Form 1:811
31. Charmetant A, Orliac J, Vidal-Sallé E, Boisse P (2012) Compos Sci Technol 72:1352

32. Ben Boubaker B, Haussy B, Ganghoffer JF (2007) Compos B Eng 38(4):498

33. Potter K (2002) Compos A Appl Sci Manuf 33(1):63

34. ten Thije R, Akkerman R, Huétink J (2007) Comput Methods Appl Mech Eng 196(33-34):3141

35. Rozant O, Bourban PE, Manson JA (2000) Compos A Appl Sci Manuf 31(11): 1167

36. Page J, Wang J (2000) Compos Sci Technol 60(7):977

37. Page J, Wang J (2002) Finite Elem Anal Des 38(8):755

38. Sun H, Pan N (2005) Compos Struct 67(3):317

39. Lomov S, Truong T, Chi Verpoest I, Peeters T, Roose D, Boisse P, Gasser A (2003) Int J Form Process 6(3-4):413

40. Zhu B, Yu T, Tao X (2007) Compos A Appl Sci Manuf 38(8): 1821

41. Zhu B, Yu TX, Teng J, Tao XM (2009) J Compos Mater 43(2): 125

42. Kawabata S, Niwa M, Kawai H (1973) J Text Inst 64(2):62

43. Naik N, Tiwari S, Kumar R (2003) Compos Sci Technol 63(5): 609

44. Lomov S, Verpoest I (2006) Compos Sci Technol 66(7-8):919

45. Xue P, Cao J, Chen J (2005) Compos Struct 70(1):69

46. King M, Jearanaisilawong P, Socrate S (2005) Int J Solids Struct 42(13):3867

47. Boisse P, Gasser A, Hagège B, Billoët J (2005) J Mater Sci 40:5955

48. Badel P, Vidal-Sallé E, Boisse P (2007) Comput Mater Sci 40(4):439

49. Badel P, Vidal-Sallé E, Maire E, Boisse P (2008) Compos Sci Technol 68(12):2433

50. Badel P, Gauthier S, Vidal-Sallé E, Boisse P (2009) Compos A Appl Sci Manuf 40(8):997

51. Lin H, Long A, Sherburn M, Clifford M (2008) Int J Mater Form 1:899

52. Durville D (2008) ArXiv e-prints

53. Khan M, Mabrouki T, Vidal-Sallé E, Boisse P (2010) J Mater Proc Technol 210:378

54. Zouari B, Daniel JL, Boisse P (2006) Comput Struct 84:351

55. Hamila N, Boisse P (2008) Compos B Eng 39(6):999

56. Yu X, Cartwright B, McGuckin D, Ye L, Mai YW (2006) Compos A Appl Sci Manuf 37(5):790

57. ten Thije R, Akkerman R (2008) Compos A Appl Sci Manuf 39(7): 1167

58. Wiggers J, Long A, Harrison P, Rudd C (2003) 6th international ESAFORM conference on material forming, pp 851-854

59. Cai Z, Gutowski T (1992) J Compos Mater 26(8):1207

60. Bel S, Hamila N, Boisse P, Dumont F (2012) Compos A Appl Sci Manuf 43:2269

61. Bel S, Boisse P, Dumont F (2012) Appl Compos Mater 19(34):513

62. Hofstee J, van Keulen F (2001) Compos Struct 54(2-3): 179

63. Durville D (2005) J Mater Sci 40:5941

64. Durville D (2007) 18ème Congrès Français de Mécanique. Grenoble

65. Durville D (2007) Micro-symposium on finite element modelling of textiles and textile composites, Saint-Petersbourg

66. Durville D (2010) Int J Mater Form 3:1241 